\title{
RELICS: High-resolution Constraints on the Inner Mass Distribution of the $z=0.83$ Merging Cluster RXJ0152.7-1357 from Strong Lensing
}

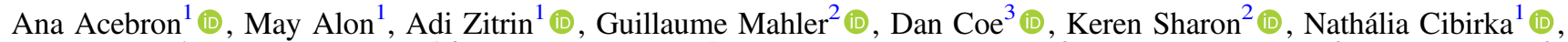 \\ Maruša Bradač ${ }^{4}$ (D) Michele Trenti ${ }^{5,6}$ (D) , Keiichi Umetsu ${ }^{7}$ (D), Felipe Andrade-Santos ${ }^{8}$ (D), Roberto J. Avila ${ }^{3}$, Larry Bradley ${ }^{3}$ (D), \\ Daniela Carrasco $^{5}$ (D), Catherine Cerny ${ }^{2}$, Nicole G. Czakon ${ }^{7}$, William A. Dawson ${ }^{9}$ (D), Brenda Frye ${ }^{10}$, Austin T. Hoag ${ }^{4}$, \\ Kuang-Han Huang $^{4}$ (D), Traci L. Johnson ${ }^{2}$ (D), Christine Jones ${ }^{8}$, Shotaro Kikuchihara ${ }^{11}$, Daniel Lam ${ }^{12}$ (D), Rachael C. Livermore ${ }^{5}$ (D), \\ Lorenzo Lovisari $^{8}$, Ramesh Mainali ${ }^{10}$ (D) Pascal A. Oesch $^{13}$ (D), Sara Ogaz ${ }^{3}$, Masami Ouchi $^{11,14}$ (D), Matthew Past ${ }^{2}$, \\ Rachel Paterno-Mahler ${ }^{2}$ (D), Avery Peterson ${ }^{2}$, Russell E. Ryan ${ }^{3}$, Brett Salmon ${ }^{3}$ (D), Irene Sendra-Server ${ }^{15,16}$, Daniel P. Stark ${ }^{10}$, \\ Victoria Strait ${ }^{4}$ (D), Sune Toft ${ }^{17}$ (iD), and Benedetta Vulcani ${ }^{5,18}$ (iD \\ ${ }^{1}$ Physics Department, Ben-Gurion University of the Negev, P.O. Box 653, Be'er-Sheva 8410501, Israel; anaacebronmunoz@gmail.com \\ ${ }^{2}$ Department of Astronomy, University of Michigan, 1085 South University Avenue, Ann Arbor, MI 48109, USA \\ ${ }^{3}$ Space Telescope Science Institute, 3700 San Martin Drive, Baltimore, MD 21218, USA \\ ${ }^{4}$ Department of Physics, University of California, Davis, CA 95616, USA \\ ${ }^{5}$ School of Physics, University of Melbourne, VIC 3010, Australia \\ ${ }^{6}$ Australian Research Council, Centre of Excellence for All Sky Astrophysics in 3 Dimensions (ASTRO 3D), Australia \\ ${ }^{7}$ Institute of Astronomy and Astrophysics, Academia Sinica, PO Box 23-141, Taipei 10617, Taiwan \\ ${ }^{8}$ Harvard-Smithsonian Center for Astrophysics, 60 Garden Street, Cambridge, MA 02138, USA \\ ${ }^{9}$ Lawrence Livermore National Laboratory, P.O. Box 808 L-210, Livermore, CA, 94551, USA \\ ${ }^{10}$ Department of Astronomy/Steward Observatory, University of Arizona, 933 N Cherry Avenue, Tucson, AZ. 85716 USA \\ ${ }^{11}$ Institute for Cosmic Ray Research, The University of Tokyo, 5-1-5 Kashiwanoha, Kashiwa, Chiba 277-8582, Japan \\ ${ }^{12}$ Leiden Observatory, Leiden University, NL-2300 RA Leiden, The Netherlands \\ ${ }^{13}$ Geneva Observatory, University of Geneva, Ch. des Maillettes 51, 1290 Versoix, Switzerland \\ ${ }^{14}$ Kavli Institute for the Physics and Mathematics of the Universe (Kavli IPMU, WPI), The University of Tokyo, Chiba 277-8582, Japan \\ ${ }^{15}$ American School of Warsaw, Warszawska 202, 05-520 Bielawa, Poland \\ ${ }^{16}$ Department of Theoretical Physics, University of Basque Country UPV/EHU, E-48080 Bilbao, Spain \\ ${ }^{17}$ Cosmic Dawn Center, Niels Bohr Institute, University of Copenhagen, Juliane Maries Vej 30, København, DK-2100, Denmark \\ ${ }^{18}$ INAF, Osservatorio Astronomico di Padova, Vicolo Osservatorio 5, I-35122 Padova, Italy \\ Received 2018 October 18; revised 2019 February 21; accepted 2019 February 25; published 2019 April 1
}

\begin{abstract}
Strong gravitational lensing (SL) is a powerful means of mapping the distribution of dark matter. In this work, we perform an SL analysis of the prominent X-ray cluster RXJ0152.7-1357 ( $z=0.83$, also known as CL0152.7-1357) in Hubble Space Telescope images, taken in the framework of the Reionization Lensing Cluster Survey (RELICS). On top of a previously known $z=3.93$ galaxy multiply imaged by RXJ0152.7-1357, for which we identify an additional multiple image, guided by a light-traces-mass approach, we identify seven new sets of multiply imaged background sources lensed by this cluster, spanning the redshift range [1.79-3.93]. A total of 25 multiple images are seen over a small area of $\sim 0.4 \mathrm{arcmin}^{2}$, allowing us to put relatively high-resolution constraints on the inner matter distribution. Although modestly massive, the high degree of substructure, together with its very elongated shape, makes RXJ0152.7-1357 a very efficient lens for its size. This cluster also comprises the third-largest sample of $z \sim 6-7$ candidates in the RELICS survey. Finally, we present a comparison of our resulting mass distribution and magnification estimates with those from a Lenstool model. These models are made publicly available through the Mikulski Archive for Space Telescopes.
\end{abstract}

Key words: galaxies: clusters: individual (RXJ0152.7-1357, CL0152.7-1357) - galaxies: high-redshift gravitational lensing: strong

\section{Introduction}

Colliding or merging galaxy clusters are unique laboratories that cannot only shed light on structure formation (Peebles et al. 1989; Planck Collaboration et al. 2016), galaxy evolution (Boselli \& Gavazzi 2006; Deshev et al. 2017), and scaling relations (Poole et al. 2007; Krause et al. 2012) of clusters during such events, but can also put important and unique constraints on the self-interaction cross section of the elusive dark matter (DM; Clowe et al. 2006; Bradač et al. 2008; Merten et al. 2011; Dawson et al. 2012).

Thanks to recent extensive observing surveys with the Hubble Space Telescope (HST; Postman et al. 2012; Lotz et al. 2017; Coe et al. 2019), a myriad of clusters at relatively low and intermediate redshifts are analyzed in great detail. Strong gravitational lensing (SL) is one of the most valuable tools for gaining insight into the distribution of DM in the core of the cluster. SL has proven to provide a determination of the total mass distribution of galaxy clusters at a percent-level precision (e.g., Richard et al. 2010; Grillo et al. 2015; Jauzac et al. 2015; Johnson \& Sharon 2016; Limousin et al. 2016; Monna et al. 2017; Cerny et al. 2018), in addition to allowing us to probe the early universe, because background galaxies are magnified by the lens (Richard et al. 2008; Zheng et al. 2012; Coe et al. 2013; Atek et al. 2015; Bouwens et al. 2017; Livermore et al. 2017; Hashimoto et al. 2018). However, only a few cases of massive merging galaxy clusters at higher redshifts, namely close to $z \sim 1$ or above, have been extensively studied (Della Ceca et al. 2000; Maughan et al. 2003; Jee et al. 2005b; Coogan et al. 2018; Paterno-Mahler et al. 2018; Khullar et al. 
2019). RXJ0152.7-1357 (also known as CL0152.7-1357 and referred to as RXJ0152 hereafter), at R.A $=1^{\mathrm{h}} 52^{\mathrm{m}} 40^{\mathrm{s}}$, decl. $=-13^{\circ} 57^{\prime} 19^{\prime \prime}$, constitutes one of these well-studied laboratories, yet it has lacked a full strong-lensing analysis until recently. ${ }^{19}$

This cluster was detected by the ROSAT Deep Cluster Survey (Rosati et al. 1998), the Wide Angle ROSAT Pointed Survey (WARPS; Ebeling et al. 2000), and the Serendipitous High-Redshift Archival ROSAT Cluster Survey (SHARC; Romer et al. 2000) as an extended source with a double core structure, as well as being among the most X-ray-luminous, massive merging clusters known at a redshift $>0.55$ (Della Ceca et al. 2000). RXJ0152 was also targeted with 14 other distant cluster candidates with the Low-Resolution Imaging Spectrograph with the Keck Telescope in the framework of the WARPS survey. The redshift of six galaxies close to the X-ray peak provided a cluster redshift of $z=0.8325$ (Ebeling et al. 2000). Subsequent X-ray studies with BeppoSAX (Della Ceca et al. 2000) and Chandra (Maughan et al. 2003; Huo et al. 2004) found RXJ0152 to consist of two main, gravitationally bound, massive and X-ray-luminous subclumps, at a projected distance of $730 \mathrm{kpc}$, and to probably be in the early stages of a massive merging process. The X-ray temperature of the whole cluster was found to be $\sim 6.5_{-1.3}^{+1.7} \mathrm{keV}$.

RXJ0152 was also targeted through the Sunyaev-Zeldovich effect (SZ) with the Berkeley-Illinois-Maryland Association millimeter interferometer (Joy et al. 2001). The authors determined its total mass to be $\sim 2 \times 10^{14} \mathrm{~h}_{100}^{-1} M_{\odot}$ within a $65^{\prime \prime}$ radius, consistent with the values inferred from the X-ray temperature measurements.

Extensive spectroscopic studies on this cluster that followed (Demarco et al. 2005; Girardi et al. 2005; Jørgensen et al. 2005) enabled the authors to characterize in detail the dynamical properties of this cluster, embedded in a larger-scale filamentary structure of the cosmic web (Tanaka et al. 2006). Dynamical studies confirmed the picture of an irregular mass distribution where cluster galaxies were observed to form substructures coinciding with those in the extended X-ray emission. These studies also indicated that the two main clumps are most likely bound and currently undergoing a merging event.

Further insights on the overall mass distribution of RXJ0152 were inferred from weak lensing (WL) studies (Huo et al. 2004; Jee et al. 2005a) thanks to the high resolution of the Advanced Camera for Surveys (ACS) observations. The WL mass estimates at a $65^{\prime \prime}$ radius were found to be in good agreement with previous results from X-ray and SZ observations. Interestingly, when comparing the WL mass reconstruction with the X-ray morphology from Chandra and optical observations, Maughan et al. (2003) and Jee et al. (2005a) noted a displacement between both the luminous and dark mass distribution and the X-ray centroids, where cluster galaxies and mass clumps seemed to lead the X-ray peaks (tracing the intracluster medium, slowed down by ram pressure). The fact that similar offsets are observed in other wellknown merging clusters (Markevitch et al. 2002; Clowe et al. 2006) further strengthens the merger scenario in RXJ0152.

In brief, previous multiprobe (X-ray, optical, SZ, dynamics, and WL) studies of RXJ0152 all characterized this system as highly unrelaxed and presenting a complex morphology, composed of a large number of subhalos.

\footnotetext{
19 We note that through RELICS, a Lenstool model was previously made available through MAST, but we present in this work the first published full SL model of the cluster.
}

In the central region of the cluster, where the SL features are seen, Umetsu et al. (2005) found the first multiple-image system, a $z=3.93$ galaxy lensed by the NE clump into three images, which allowed them to study the lensed galaxy in detail and construct a simple symmetric mass model for the NE clump (with some priors drawn from previous mass estimates from WL by Jee et al. 2005a). However, having only one multiple-image system usually allows one to only assess the enclosed mass within the system's effective Einstein radius, rather than to actually constrain the overall mass distribution and profile of the cluster.

In this work, we have taken advantage of the recent Reionization Lensing Cluster Survey (RELICS) observations (e.g., Cerny et al. 2018; Coe et al. 2019) to revisit the SL modeling of RXJ0152, the third-highest-redshift cluster of the sample. We identify various additional sets of multiple images spread throughout the central clumps, allowing us to constrain in detail the inner mass distribution of the cluster using two well-known modeling tools.

A major goal of the RELICS survey is to detect a large sample of high-redshift (Salmon et al. 2017), magnified galaxies. SL models for high-redshift clusters are of great interest as the cluster lensing power increases significantly with source redshift compared to lower-redshift counterparts (Zitrin et al. 2013a). Indeed, RXJ0152 presents the third-largest sample of high- $z(z \gtrsim 6)$ candidates from the RELICS cluster sample (Salmon et al. 2017), making the SL models presented here crucial for accurately determining the properties of these high-redshift candidates as well as translating the sample of candidates into a galaxy luminosity function.

This work is organized as follows. In Section 2 we briefly describe the observations. These were used to identify multiple images considered for the SL analysis, presented in Section 3. The results are presented and discussed in Section 4. Finally, the work is summarized in Section 5. Throughout we assume a $\Lambda \mathrm{CDM}$ cosmology with $\Omega_{\mathrm{m} 0}=0.3, \quad \Omega_{\Lambda} \quad 0=0.7, \quad H_{0}=$ $100 h \mathrm{~km} \mathrm{~s}^{-1} \mathrm{Mpc}^{-1}$, with $h=0.7$, where $1^{\prime \prime}=7.71 \mathrm{kpc}$ at the redshift of RXJ0152.

\section{Data and Observations}

\subsection{Imaging}

The cluster analyzed in the present work is part of the RELICS cluster sample (PI: D. Coe; Coe et al. 2019). The RELICS program has targeted 41 mainly SZ-selected massive clusters (including several other criteria; see Cerny et al. 2018, or Coe et al. 2019, for more details) to efficiently search for magnified high-redshift galaxies in time for spectroscopic follow-up with the James Webb Space Telescope (Salmon et al. 2017). Given that some HST archival observations already existed for RXJ0152 (program 9290 and follow-up observations searching for supernovae in programs 10493 and 10793), RELICS completed the observations needed to make this cluster a coherent part of the RELICS sample. In total, including the previous observations, RXJ0152 has been observed for a total of three orbits with the ACS (in the F435W, F625W, F775W, and F850LP bands), two orbits with the Wide Field Camera 3 (WFC3/IR in the F105W, F125W, F140W, and F160W bands), and $30 \mathrm{hr}$ per band of each of the Spitzer-IRAC channels (PI: M. Bradac, PI: Soifer). In this work, we used the reduced HST images and photometric source catalogs generated with SExtractor (Bertin \& Arnouts 1996) in 
dual-image mode from the final drizzled 0."06 images. Bayesian photometric redshifts (hereafter $z_{\text {phot }}$ ) were derived using the Bayesian Photometric Redshift program (BPZ; Benítez 2000; Benítez et al. 2004; Coe et al. 2006) from seven HST band imaging data (both from RELICS observations and $H S T$ archival data). These data products are available for the community through the Mikulski Archive for Space Telescopes (MAST). ${ }^{20}$

\subsection{Spectroscopic Observations}

The cluster was observed with LDSS3-C ${ }^{21}$ on the Magellan/ Clay telescope on 2017 July 27 (University of Michigan allocation, PI: Sharon). The seeing ranged between $0 . " 5$ and 0 !. 7 with thin clouds throughout the night. The data were obtained with the VPH-ALL grism $(4250 \AA<\lambda<10000 \AA)$. The spectra were reduced using the standard COSMOS routines (Dressler et al. 2011; Oemler et al. 2017). A full description of spectroscopic follow-up will be presented in a forthcoming paper (R. Mainali et al. 2019, in preparation). We measure two secure redshifts in this field, both from detection of $\operatorname{Ly} \alpha$. An image of system 1 at 1:52:45.358, -13:57:07.75 confirmed the redshift previously measured by Umetsu et al. (2005), $z_{\mathrm{spec}}=3.930$, and a galaxy at 1:52:39.566, $-13: 58: 37.11$, $z_{\text {spec }}=3.611$.

\section{Lens Model}

\subsection{The LTM Pipeline}

We perform the SL analysis using the LTM method by Broadhurst et al. (2005) and Zitrin et al. (2009). The light-tracesmass (LTM) model has proven to be a powerful method to both identify new multiple images and constrain the cluster mass distribution (e.g., Merten et al. 2011; Zitrin et al. 2015; Frye et al. 2019). The LTM pipeline has been adopted as well to model other RELICS clusters (see Acebron et al. 2018; Cibirka et al. 2018). We give here a brief overview of the pipeline, but we refer the reader to these recent papers for further details.

Our method relies on the assumption that the underlying DM distribution in the cluster is traced by the distribution of the luminous component, namely, cluster galaxies. This brings to a minimum the number of free parameters needed to generate a mass model, while still possessing sufficient flexibility to describe the underlying mass distribution. The position and source redshift (where available) of multiple images are used as constraints for the SL modeling.

We start constructing a mass model by identifying cluster members, following the red-sequence method (Gladders \& Yee 2000). We use the magnitudes measured from the F606W and F814W filters to draw a color-magnitude diagram and consider only galaxies down to $24 \mathrm{AB}$ within \pm 0.3 mag of the sequence. We then apply several criteria to exclude stars from our selection: we consider objects with magnitudes fainter than $17 \mathrm{AB}$ with a cutoff value for the stellarity index of $<0.95$ and rely as well on the help of a size-magnitude relation, plotting the FWHM versus the F814W magnitude in which stars occupy a specific region of the parameter space. An important step is a subsequent visual inspection of the selected cluster members, where we discard further interloping galaxies (bright foreground galaxies, for instance) or artifacts (such as faint and

\footnotetext{
20 https://archive.stsci.edu/prepds/relics/

21 http://www.lco.cl/telescopes-information/magellan/ instruments/ldss-3
}

diffuse objects). We also used the delivered photometric catalog, which includes photometric redshift estimates from BPZ, to check that all selected cluster members were within $z_{\text {phot }} \pm 0.1$ of the mean redshift of the cluster. Finally, we also compared our selection with previous publicly available spectroscopic catalogs from Demarco et al. (2005, 2010). In the most central regions of the cluster, apart from the brightest central cluster members, a large number of galaxies that appear to be red-sequence cluster members were lacking a spectroscopic confirmation, so we chose to rely on the red-sequence method for the rest of the analysis.

Once a final list of cluster members is constructed, each cluster member is then parameterized by a symmetric powerlaw surface mass-density distribution, scaling linearly in amplitude with luminosity (for some galaxies, ellipticity or other scaling relations can be introduced; see below). The power-law exponent is the first free parameter of the model and is the same for all galaxies. The DM distribution, as is assumed in our method, will follow the luminous component as well but is smoothed with a 2D Gaussian whose width is the second free parameter of the model. Both components are then coadded, with their relative weight being the third free parameter. The fourth free parameter refers to the overall normalization. Our method allows for further flexibility by adding a two-parameter external shear (which introduces ellipticity to the magnification map) parameterized by its amplitude and its position angle, bringing a total of six basic free parameters. Finally, to better reproduce the observations, other parameters can be introduced, such as the weight of the brightest cluster galaxy (BCG) and its ellipticity, position angle, or redshift of background sources, which can be optimized by the pipeline.

The goodness of the fit is assessed using a $\chi^{2}$ criterion during the minimization, which quantifies the quality of reproduction of multiple-image positions in the image plane, given by

$$
\chi^{2}=\sum_{i=1}^{n} \frac{\left(x_{i}^{\mathrm{pred}}-x_{i}^{\mathrm{obs}}\right)^{2}+\left(y_{i}^{\mathrm{pred}}-y_{i}^{\mathrm{obs}}\right)^{2}}{\sigma_{i}^{2}},
$$

where the the difference between the model-predicted $x_{i}^{\text {pred }}$, $y_{i}^{\text {pred }}$ and observed positions $x_{i}^{\text {obs }}, y_{i}^{\text {obs }}$ of the multiple images is weighted by the observational uncertainty $\sigma_{i}$ (assumed here to be $0 . " 5$ for all multiple images).

Independently, we can also assess the goodness of fit of a model with the rms between the observed and model-predicted positions of the multiple images in the image plane, which can written as follows:

$$
\mathrm{rms}=\sqrt{\frac{1}{N_{\mathrm{img}}} \sum_{i=1}^{n}\left(\left(x_{i}^{\mathrm{pred}}-x_{i}^{\mathrm{obs}}\right)^{2}+\left(y_{i}^{\mathrm{pred}}-y_{i}^{\mathrm{obs}}\right)^{2}\right)},
$$

where $N_{\text {img }}$ is the total number of images.

\subsection{Identification of Multiple Images}

In an iterative way and starting with a simple initial model, our method predicts both the shape and orientation of multiply imaged candidates by sending them to the source plane and back to the image plane using the lens equation (namely $\beta=\theta-\alpha$, where $\beta$ is the angular source position, $\theta$ the observed image position, and $\alpha$ the so-called reduced (i.e., scaled) deflection angle, in this case given by the initial model). Using these predictions, we thus can identify new multipleimage families based on similar colors, morphology, and 


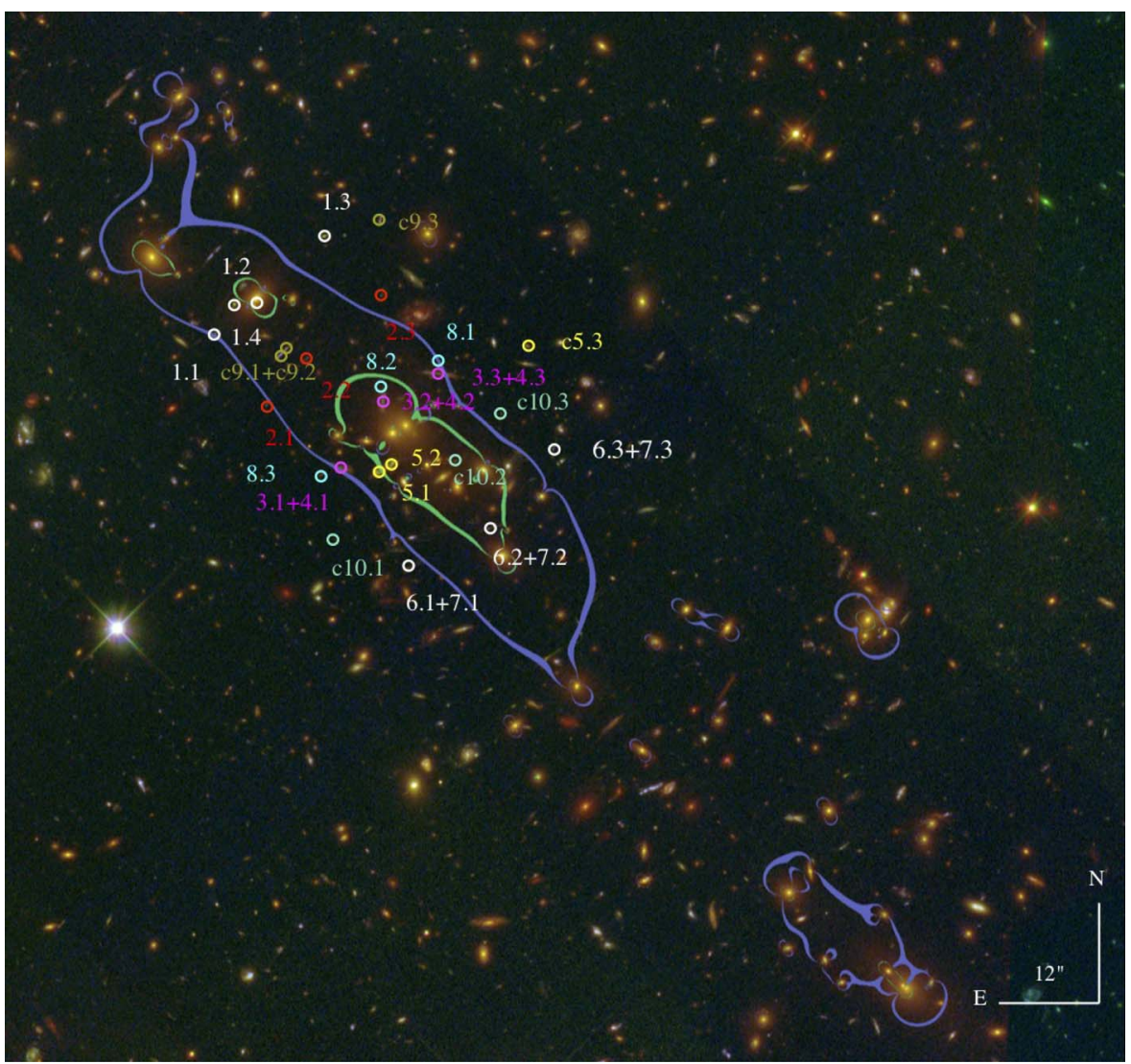

Figure 1. Color-composite image of RXJ0152. Image was created using the HST/ACS passbands F435W (blue), a combination of F606W+F814W (green), and a combination of the $H S T / \mathrm{WFC} 3 \mathrm{IR}$ passbands F105W $+\mathrm{F} 125 \mathrm{~W}+\mathrm{F} 140 \mathrm{~W}+\mathrm{F} 160 \mathrm{~W}$ (red). The resulting critical curves from our best-fit model are displayed for a source at $z \sim 2.0$ (green) and $z \sim 9.0$ (violet). Multiple images (color coded to ease their identification) are labeled according to Table 1.

symmetry, allowing us to refine the initial model. In this study, we only use as constraints the position of multiply imaged systems that we consider secure (i.e., those whose agreement with the model prediction, internal details, similar colors, and symmetry leave essentially no doubt these are images of the same source).

The first multiply imaged system used to constrain the mass model was reported by Umetsu et al. (2005), who measured a spectroscopic redshift of 3.93 with the Faint Object Camera and Spectrograph on the Subaru telescope. This background galaxy is lensed by the NE clump into three multiple images appearing as a greenish galaxy on the HST composite image in Figure 1. We report, however, an additional image in the system, image 1.4, which lies next to a nearby cluster member. This is the only system having a redshift spectroscopically confirmed. In addition, we have identified seven other multiply imaged systems, displayed in Figure 1 and listed in Table 1. System 2 comprises three multiple images that have similar colors in the HST composite image. Systems 3 and 4, with three multiple images each, show an arc-shaped image in the central region of the cluster. Due to the difference in color between the two ends of the arc, we mark them as two different systems, supplying two sets of constraints to the model. System 5 consists of three multiple images: images 5.1 and 5.2 are stretched into an arc shape, with two bright emission knots, appearing light green in a composite color image as in Figure 1. A candidate counterimage c5.3 sits on the other side of the opposite critical curve. As seen in Table 1, the redshift of this system is not well constrained with the LTM optimization. We use a predicting tool to delens one image of the system to the source plane and back to the image plane to compare the model-predicted and observed location and orientation reproduction. Our best-fit LTM model prefers a higher redshift (in the redshift range 2.5-3.1), in good agreement with the Lenstool and BPZ estimations.

The three multiple images making up system 6 and system 7 appear as two bright peaks with similar colors, lying next to each other. Finally, system 8 has three multiple images that appear as a bright peak with a long tail. All multiple images are marked in Figure 1, and their reproduction by our best-fit model is shown in Figure 5. Our model only predicts two additional, fainter multiple images for system 5 . Other, less secure, multiply imaged systems predicted by our SL model are reported in Table 1 as candidates. System c9 appears as three images, one of them being a pink arc, with several emission knots (images c9.1 and c9.2). System c10 comprises three green images and is considered as a candidate because few nearby objects are similar in terms of colors and morphology, diminishing the reliability of our candidate identification. Therefore, we chose not to include these counterimages in the modeling and only refer to them as possible candidates.

We find that the best SL model for RXJ0152 is obtained when not considering any galaxy as predominant (i.e., a BCG) because its structure shows a very elongated cluster with no clear central, predominant region. Typically, with the LTM formalism, the BCG is found to contain more mass with respect to its light compared to other members, and we therefore usually allow its mass-to-light ratio to vary, while we found this was not needed for the modeling of RXJ0152. We also do 
Table 1

Multiple Images and Candidates for RXJ0152.7-1357

\begin{tabular}{|c|c|c|c|c|c|c|c|c|}
\hline Arc ID & $\begin{array}{l}\text { R.A. } \\
\text { (deg) }\end{array}$ & $\begin{array}{l}\text { Decl. } \\
\text { (deg) }\end{array}$ & $z_{\text {phot }}\left[z_{\min }-z_{\max }\right]^{\mathrm{a}}$ & $z_{\text {spec }}$ & $z_{\text {model }}^{\text {LTM }}[68 \% \text { C.I. }]^{\mathrm{b}}$ & $z_{\text {model }}^{\text {Lenstool }}[68 \% \text { C.I. }]^{\mathrm{c}}$ & Comments & Individual rms (") $)^{\mathrm{d}}$ \\
\hline 1.1 & 28.189012 & -13.952162 & $3.79[3.70-3.89]$ & $3.93^{\mathrm{e}}$ & $3.93^{\mathrm{f}}$ & 3.93 & $\cdots$ & 1.63 \\
\hline 1.2 & 28.188295 & -13.951124 & $3.77[3.61-3.91]$ & $"$ & $"$ & " & $\cdots$ & 1.27 \\
\hline 1.3 & 28.185218 & -13.948862 & $3.85[3.77-3.94]$ & ” & ” & ” & $\ldots$ & 1.35 \\
\hline 1.4 & 28.187603 & -13.951152 & $\ldots$ & $"$ & ” & ” & close to a cluster member & 0.38 \\
\hline 2.1 & 28.187214 & -13.954498 & $2.81[2.42-3.14]$ & $\cdots$ & $3.80[3.03-4.23]$ & $3.58[2.46-3.67]$ & $\ldots$ & 0.87 \\
\hline 2.2 & 28.185771 & -13.952901 & $3.25[2.96-3.52]$ & $\ldots$ & $"$ & " & $\ldots$ & 0.26 \\
\hline 2.3 & 28.183267 & -13.950841 & $0.66[0.22-3.13]$ & $\cdots$ & $"$ & " & $\cdots$ & 0.85 \\
\hline 3.1 & 28.184847 & -13.956517 & $1.80[1.54-1.98]$ & $\cdots$ & $1.98[1.67-2.01]$ & $1.58[1.50-1.68]$ & $\cdots$ & 0.38 \\
\hline 3.2 & 28.183187 & -13.954174 & $1.06[1.00-1.18]$ & $\ldots$ & " & $"$ & $\ldots$ & 0.96 \\
\hline 3.3 & 28.181607 & -13.953402 & $0.27[0.03-0.46]$ & $\ldots$ & $"$ & " & $\ldots$ & 0.14 \\
\hline 4.1 & 28.184489 & -13.956624 & 1.79 [1.61-1.89] & $\ldots$ & $1.97[1.68-2.01]$ & $1.58[1.50-1.70]$ & $\ldots$ & 0.20 \\
\hline 4.2 & 28.183217 & -13.954531 & $\ldots$ & $\ldots$ & $"$ & " & $\ldots$ & 1.15 \\
\hline 4.3 & 28.181020 & -13.95347 & 1.79 [1.55-1.92] & $\ldots$ & ” & " & $\ldots$ & 0.68 \\
\hline 5.1 & 28.183455 & -13.956739 & $3.27[3.16-3.35]$ & $\ldots$ & $1.79[1.60-10.83]$ & $2.26[2.02-2.63]$ & $\ldots$ & 0.36 \\
\hline 5.2 & 28.182859 & -13.956402 & $0.21[0.16-0.50]$ & $\ldots$ & $"$ & $"$ & $\ldots$ & 0.85 \\
\hline c5.3 & 28.178268 & -13.952506 & $3.13[2.94-3.32]$ & $\cdots$ & ” & ” & $\cdots$ & $\cdots$ \\
\hline 6.1 & 28.182379 & -13.959926 & $3.01[0.09-3.26]$ & $\ldots$ & $3.00[2.39-3.10]$ & $2.06[1.83-2.43]$ & $\ldots$ & 0.55 \\
\hline 6.2 & 28.179398 & -13.958503 & $3.11[2.49-3.38]$ & $\ldots$ & $"$ & " & $\ldots$ & 0.46 \\
\hline 6.3 & 28.177391 & -13.955948 & $2.79[2.51-3.06]$ & $\cdots$ & ” & " & $\cdots$ & 0.68 \\
\hline 7.1 & 28.182153 & -13.959811 & 1.15 [1.14-3.03] & $\ldots$ & $3.13[2.36-3.12]$ & $2.02[1.77-2.36]$ & $\ldots$ & 1.19 \\
\hline 7.2 & 28.179577 & -13.958584 & 1.16 [1.12-3.03] & $\cdots$ & ” & ” & $\ldots$ & 0.41 \\
\hline 7.3 & 28.177323 & -13.955818 & $2.78[2.33-3.09]$ & $\cdots$ & ” & " & $\cdots$ & 1.01 \\
\hline 8.1 & 28.181273 & -13.953073 & $2.88[2.64-3.28]$ & $\ldots$ & 2.17 [1.84-2.18] & 1.79 [1.69-1.94] & $\ldots$ & 0.49 \\
\hline 8.2 & 28.183334 & -13.954073 & $\cdots$ & $\cdots$ & ” & ” & $\cdots$ & 0.85 \\
\hline 8.3 & 28.185257 & -13.956773 & $1.91[1.48-2.38]$ & $\cdots$ & ” & ” & $\cdots$ & 0.18 \\
\hline c9.1 & 28.186812 & -13.952893 & $1.91[1.82-2.37]$ & $\cdots$ & $\sim 2.8$ & $\cdots$ & not used as constraint & $\ldots$ \\
\hline c9.2 & 28.186540 & -13.952623 & $2.51[2.32-2.63]$ & $\cdots$ & ” & $\cdots$ & $\cdots$ & $\cdots$ \\
\hline c9.3 & 28.183312 & -13.948390 & $1.70[1.59-1.79]$ & $\cdots$ & " & $\cdots$ & $\cdots$ & $\cdots$ \\
\hline $\mathrm{c} 10.1$ & 28.184906 & -13.958930 & $3.82[0.19-4.12]$ & $\cdots$ & $\sim 2.6$ & $3.25[2.91-3.67]$ & not used as constraint & $\cdots$ \\
\hline $\mathrm{c} 10.2$ & 28.180724 & -13.956335 & $\cdots$ & $\cdots$ & $"$ & $\cdots$ & $\cdots$ & $\cdots$ \\
\hline $\mathrm{c} 10.3$ & 28.179147 & -13.954763 & $3.40[2.92-3.67]$ & $\cdots$ & ” & $\cdots$ & $\cdots$ & $\cdots$ \\
\hline
\end{tabular}

Notes.

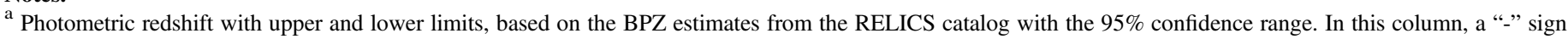
indicates an image for which its $z_{\text {phot }}$ could not be measured due to light contamination or poor signal-to-noise ratio.

${ }^{\mathrm{b}}$ Redshift prediction based on our LTM best-fit model.

${ }^{c}$ Redshift prediction based on our Lenstool best-fit model.

${ }^{\mathrm{d}}$ rms between the observed and model-predicted multiple images from our LTM best-fit model.

e Umetsu et al. (2005).

${ }^{\mathrm{f}}$ Fixed redshift for the LTM modeling.

not assign any ellipticity for the central bright galaxy. We do, however, optimize both the ellipticity and position angle of the bright cluster member of the NE clump, close to the images of system 1, which slightly improves their reproduction.

We scale our model to the spectroscopic redshift of system 1 (see Table 1) and leave the redshift of the remaining systems as free parameters to be optimized in the minimization procedure (allowing the corresponding $D_{\mathrm{LS}} / D_{\mathrm{S}}$ ratio for each system to vary by -0.3 and +0.5 ).

The optimization of the model is carried out with several thousand Markov chain Monte Carlo (MCMC) steps and includes a total of 14 free parameters when accounting, in addition, for freely optimized galaxies and source redshifts where needed.

The resulting critical curves (for a source at $z_{s}=2$ and $z_{s}=9$ ) for our final best-fit model, which has an image reproduction rms $=0$ " 84, are shown in Figure 1. The reproduction of the multiple images used as constraints in our model is shown in Figure 5 in Appendix A, and the obtained best-fit parameters are presented in Table 3 in Appendix C, which are specific to our methodology because the LTM model is not analytic.

\subsection{The Lenstool Pipeline}

RXJ0152.7-1357 was also modeled with the Lenstool ${ }^{22}$ pipeline (see Jullo et al. 2007 for further details), and model products were made publicly available by the RELICS team through MAST. In order to compare the main SL outputs between the two modeling algorithms, we revisit the Lenstool

\footnotetext{
22 https://projets.lam.fr/projects/lenstool
} 


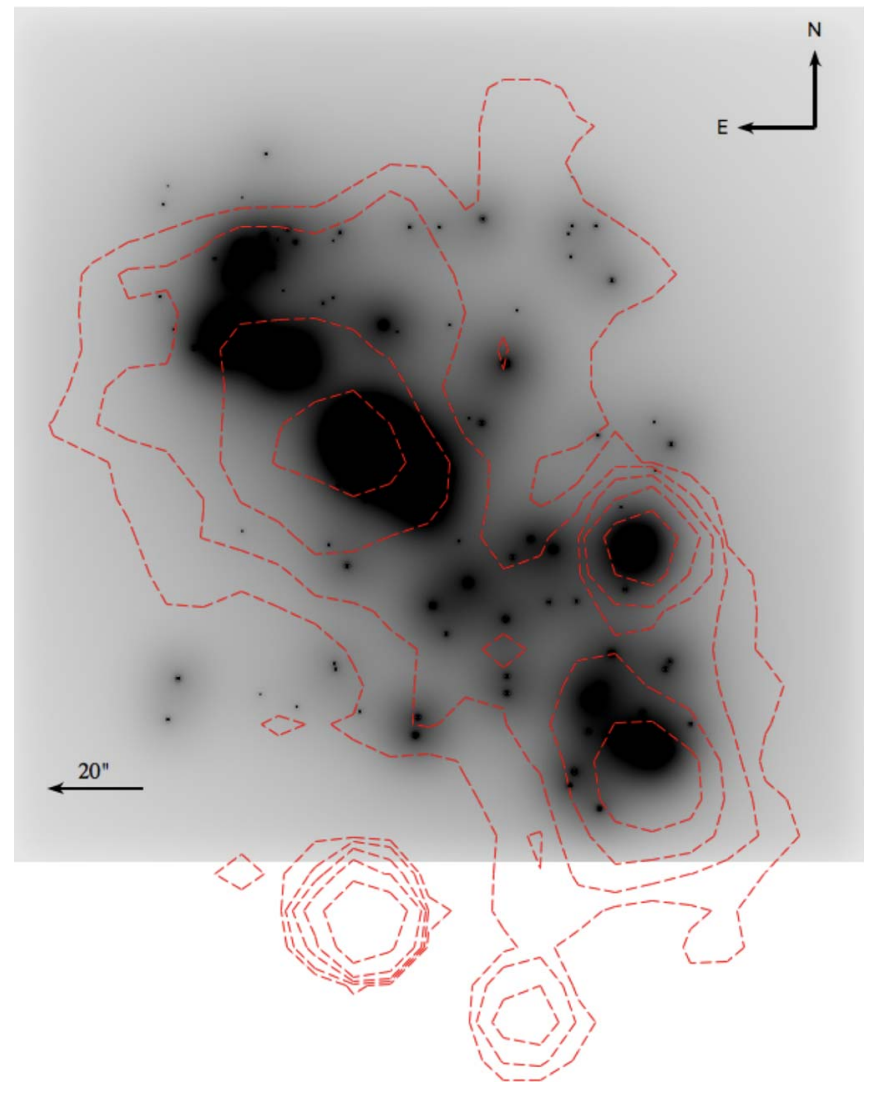

Figure 2. Left panel: convergence $\kappa$ map from our best-fit LTM model (referring to the projected surface mass density in units of the critical density for lensing $\Sigma_{\text {crit }}$ ), scaled to a source redshift of $z_{s} \sim 2.0$; overlaid are the smoothed X-ray results (red dashed contours) from previous Chandra observations.

analysis and compute a second version (V.2) using the same lensing constraints as the LTM model, except for system 9, which is also included in the Lenstool model. We provide here a comparison of the main SL outputs between the LTM and Lenstool pipelines but refer the reader to a forthcoming study for a more detailed and extensive comparison (see also Meneghetti et al. 2017; Remolina González et al. 2018 for comparison studies of different SL algorithms).

RXJ0152 is modeled using the same constraints reported in Table 1 except for system 9, which is included in the Lenstool modeling (images 9.1 and 9.2). Both the large- and small-scale halos are parameterized by a pseudoisothermal density profile (PIEMD; Kassiola \& Kovner 1993). We optimize the ellipticity, position angle, core radius, and velocity dispersion of the main large-scale halo; the central coordinates of the halo are also let free during the optimization. Moreover, the cluster member close to system 1 is modeled independently with a PIEMD profile where the core radius and velocity dispersion are optimized during the minimization procedure (i.e., not following the scaling relations). The LTM pipeline provides a hint of an additional mass in the outskirts of the cluster in the SW direction that can also be seen in the X-ray map (see Figure 2). We found that an additional large-scale clump for this SW structure improved the Lenstool fit by $\sim 0$." 1 . However, this improvement is not significant enough in terms of the Bayesian information criterion, which prefers a model not including additional free parameters to be optimized in a region with no lensing constraints.
Finally, the small-scale halos associated with cluster members are also parameterized with a PIEMD profile with a fixed core radius of $0.01 \mathrm{kpc}$, a velocity dispersion allowed to vary between 50 and $200 \mathrm{~km} \mathrm{~s}^{-1}$, a cut radius varying from 20 to $200 \mathrm{kpc}$, and following the scaling relations (Faber \& Jackson 1976). We assign the ellipticity and position angle values measured from the light distribution with SEXtractor (Bertin \& Arnouts 1996) to model the underlying DM distribution. As in the case of our modeling with LTM, the redshifts of all systems but system 1 are optimized with a flat prior.

Our resulting best-fit model from Lenstool has an rms of 0 ." 52 . The best-fit parameters are shown in Table 4 in Appendix C, and the resulting critical curves and magnification map from our bestfit model are shown in Figures 6 and 7 in Appendix B. We have also carried out a model where the galaxy halos are considered spherical (as in the LTM model). The resulting rms is 0.159 , which is very similar to our fiducial model, and their mass profiles are also equivalent within the statistical uncertainties. Finally, we have also modeled RXJ0152 without system 9 with Lenstool. This model yields an equivalent fit (with an rms of 0 . 53) in terms of best-fit parameters to that of our fiducial model, but the latter yields a more robust mass profile estimation in the inner cluster region, that is, with lower statistical uncertainties.

\section{Results and Discussion}

Both the surface mass-density distribution from our best-fit model and the mass profile are shown in Figures 2 and 3, respectively. Our SL analysis reveals, as implied by its member galaxy distribution, a highly elongated cluster in the NE-SW direction (see the $\kappa$ map in the left panel of Figure 2), composed of several clumps. We also compare the mass distribution of RXJ0152 obtained from our SL analysis to previous high-resolution X-ray observations with Chandra (ObsId 913; Ebeling et al. 2000; Maughan et al. 2003) in Figure 2 (see the dashed red contours).

While our LTM pipeline strongly follows the assumption that light traces mass, Jee et al. (2005a) showed in their WL analysis that there exists a strong correlation between both the light and mass components. However, the X-ray peaks are displaced with respect to the peaks of the mass distribution (see also Maughan et al. 2003). Together with previous X-ray (Maughan et al. 2003) and WL studies (Jee et al. 2005a), the elongated, filamentary-like structure of the SL region further supports the merging scenario.

We compute the effective Einstein radius of RXJ0152, defined as $\theta_{\mathrm{E}}=\sqrt{A / \pi}$, where $A$ refers to the area enclosed within the critical curves. Our SL analysis reveals a relatively small lens, with an effective Einstein radius of $\theta_{\mathrm{E}}\left(z_{s}=2\right)=8.5 \pm 1^{\prime \prime}$ and a corresponding enclosed mass within the critical curves of $(2.5 \pm 0.4) \times 10^{13} M_{\odot}$ (with the uncertainties typically encompassing both the statistical and systematic errors, e.g., Zitrin et al. 2015). The critical area increases significantly for higher redshift sources due to the merging of the critical curves by different clumps, as shown in Figure 1, reaching $\theta_{\mathrm{E}}\left(z_{s}=9\right)=19 \pm 2^{\prime \prime}$. We also find an Einstein radius of $\theta_{\mathrm{E}}\left(z_{s}=3.93\right)=15.2 \pm 1$." 5 , in agreement with the previous estimation from Umetsu et al. (2005).

Figure 4 shows the magnification map for a source at redshift $z_{s}=6.5$ (left panel) together with the position of the high- $z$ candidates reported by Salmon et al. (2017) within RXJ0152's field of view. Overall we find that our magnification estimation is 


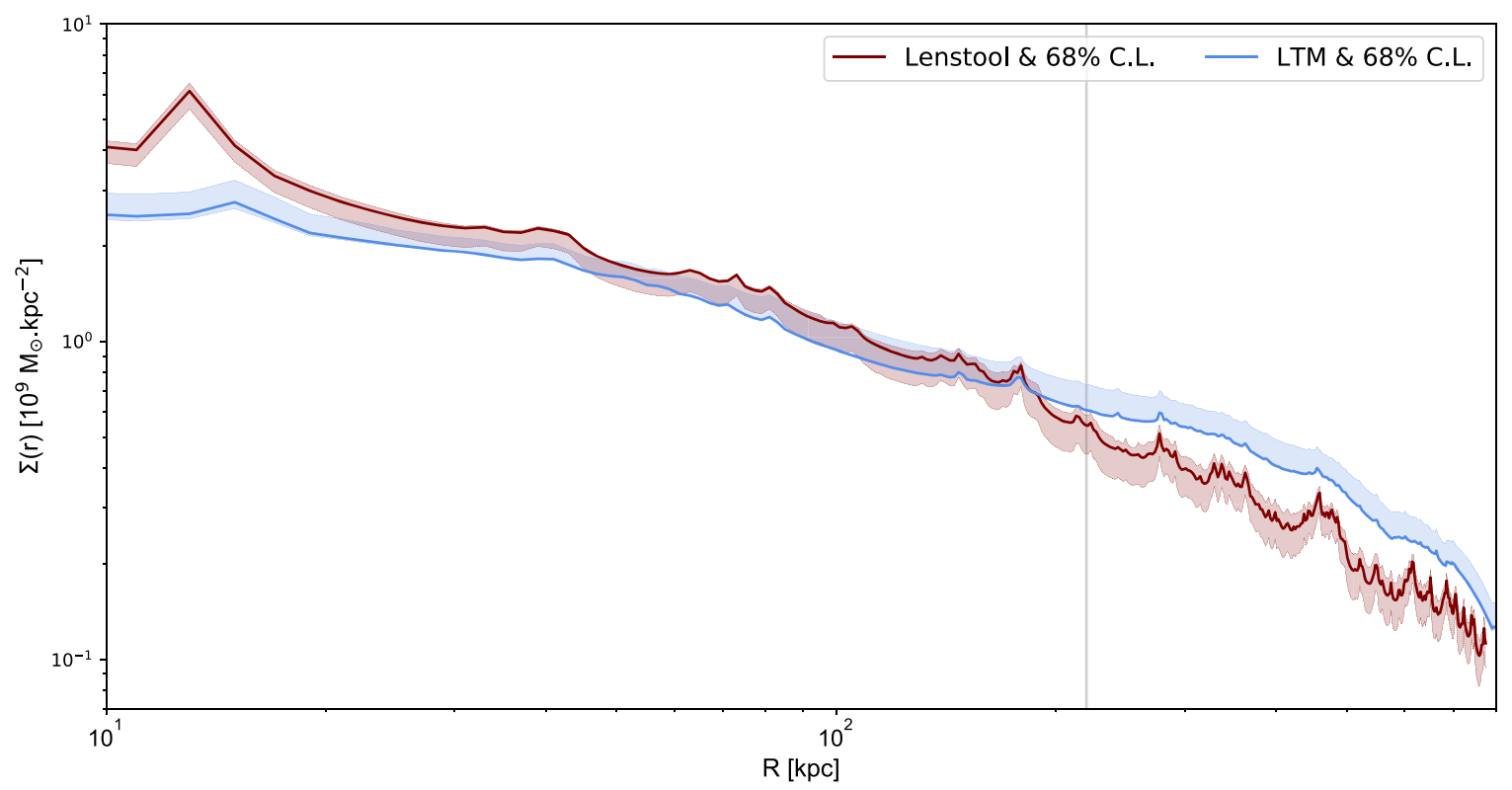

Figure 3. Mass profile computed as the mass within an annulus at a certain radius for the LTM and Lenstool models in blue and red, respectively. Both profiles are centered in one of the BCGs. The black dashed vertical line sets the radius within which we have identified multiple images.
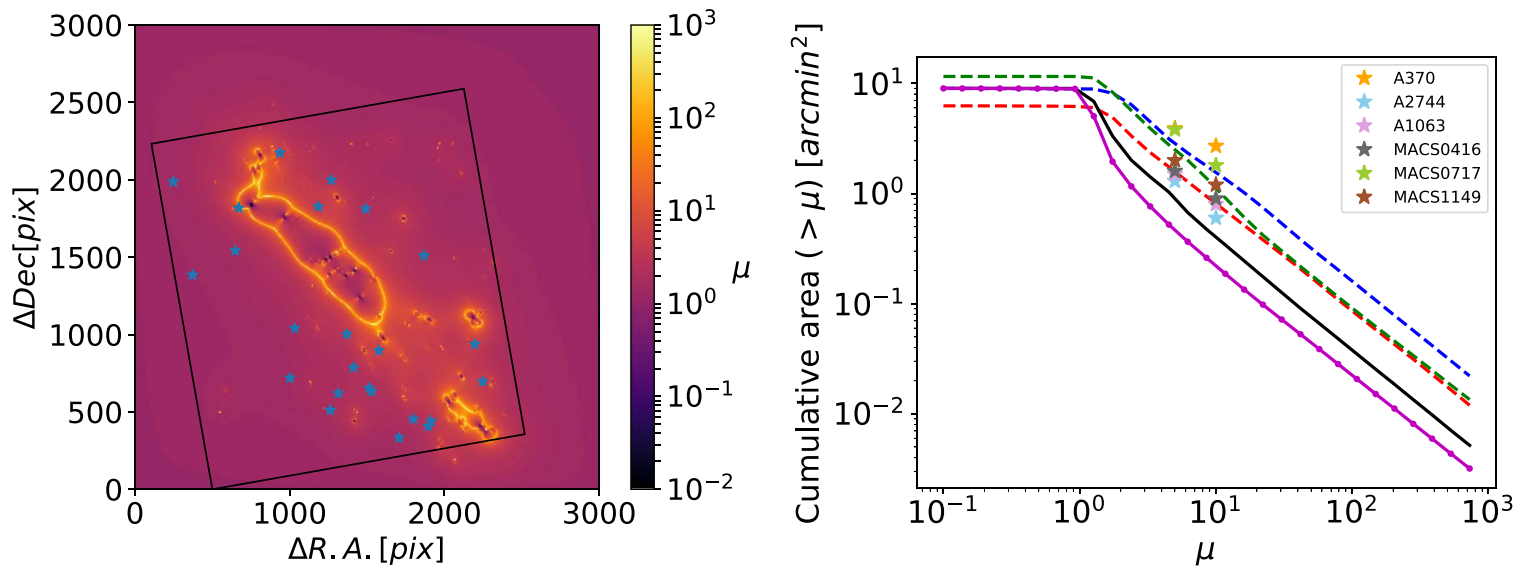

Figure 4. Left panel: magnification map from our best-fit LTM model for a source at $z_{s}=6.5$, the redshift around which the majority of the RELICS high- $z$ candidates were found in Salmon et al. (2017), pictured as blue stars. The black rectangle indicates the WFC3/IR FOV with a different roll angle to fit in all high- $z$ candidates. Right panel: we also assess the strength of RXJ0152 as a lens, comparing the cumulative area having a magnification higher than a given value for a source at $z_{s}=9.0$ (in black and magenta for our LTM and Lenstool models, respectively) with those from other known efficient RELICS SL clusters modeled with the LTM pipeline, MACS J0308.9+2645, PLCK G171.9-40.7, and AS295 (in blue, red, and green, respectively). The cumulative areas $(\mu>5$ and $\mu>10)$ for the Hubble Frontier Fields clusters are also indicated as colored stars, computed from the submitted ZITRIN-LTM-GAUSS models. The $1 \sigma$ errors are typically of the size of the star symbol.

constrained to better than $20 \%$ in at least $80 \%$ of the modeled field of view (FOV). The cumulative area magnified above a certain magnification value (which assesses the strength of the lens) at a source redshift $z_{s}=9.0$ is shown in the right panel. RXJ0152 covers a modest area of high magnification, $\sim 1.05 \operatorname{arcmin}^{2}$ for $\mu>5$ to $\sim 0.47 \operatorname{arcmin}^{2}$ for $\mu=10$, for a source at redshift $z_{s}=9.0$. The cumulative area measured for RXJ0152 is compared to other RELICS clusters that provided a large highmagnification area, MACS J0308.9+2645 and PLCK G171.940.7, presented in Acebron et al. (2018) and AS295 (see Cibirka et al. 2018). As in previous works, we also mark for reference the corresponding areas $A(\mu>5)$ and $A(\mu>10)$ for the Hubble Frontier Field (HFF) clusters (Lotz et al. 2017), computed from the ZITRIN-LTM-GAUSS models (in the full area provided for each cluster). Even though RXJ0152 is a significantly smaller (less massive) lens, its lensing strength is nearly comparable to both the typical HFF or the RELICS clusters lensing strengths from both the LTM and Lenstool models. We also point out that the LTM lensing strength is slightly greater than that from the Lenstool model, apart from the final modeling differences, because of the structure in the SW that we chose to not include in the Lenstool model due to the lack of lensing constraints in that region. This SW structure creates an additional region of high magnification in our LTM model. The high lensing efficiency of RXJ0152 is partly due to its merging state, where high-magnification regions arise between the merging subclumps or substructures projected on the plane of the sky (i.e., Torri et al. 2004; Meneghetti et al. 2007; Fedeli et al. 2010; Redlich et al. 2012; Wong et al. 2012; Cibirka et al. 2018).

A primary goal of the RELICS observations was to detect a statistically significant number of high-redshift galaxies. Salmon et al. (2017) performed an extensive photometric 
study of the 41 RELICS cluster fields, revealing 321 candidate galaxies with photometric redshifts between $z \sim 6$ and $z \sim 8$.

Particularly, the authors found large samples of such highredshift candidate galaxies in fields magnified by relatively high-redshift (i.e., close to $z \sim 1$ ), morphologically complex clusters. Indeed, for a given lens angular-diameter distance $D_{\mathrm{L}}$, the lensing signal depends on the angular-diameter distances to the source $\left(D_{\mathrm{S}}\right)$ and from the lens to the source $\left(D_{\mathrm{LS}}\right)$ as $\propto D_{\mathrm{LS}} / D_{\mathrm{S}}$. This means that for clusters at high redshift, the lensing power increases significantly with source redshift, relative to the slow increase in low-redshift clusters. For instance, the highest-redshift cluster $(z=0.972)$ for which a full strong lens model was recently published is SPT-CLJ06155746 (Paterno-Mahler et al. 2018), based on RELICS observations. Their SL analysis reveals critical curves that substantially increase from $z_{s}=1.3$ to $z=9.93$ and together with RXJ0152 presented the second- and third-largest sample of high-redshift galaxies, respectively, within any RELICS cluster's field of view. Similarly, Zitrin et al. (2013a) carried out a lensing analysis with LTM on another famous galaxy cluster ACT-CLJ0102-49151 at a similarly high redshift, $z=0.87$, known as El Gordo (and also part of the RELICS sample). The authors found that the two central clumps, each forming its own modest critical curve for a source redshift $z_{s}=2$, rapidly increase with source redshift, and the two critical regions merge into a large, elongated lens for sources at $z_{s}=9$. For such high-redshift clusters, the power to lens $z \sim 1-2$ background galaxies is small, but increases rapidly for higher redshift sources. More recently, and adopting the fully parametric SL algorithm Lenstool (Jullo et al. 2007), Cerny et al. (2018) found similar results where its Einstein radius increases from $R_{\mathrm{E}}=27.2 \pm 1$." 4 for a source at $z_{s}=3.0$ to $R_{\mathrm{E}}=40.3 \pm 2$ !" 0 at $z_{s}=9.0$ and leading to the discovery of the fourth-largest sample of high- $z$ galaxy candidates in RELICS.

The high- $z$ candidates within RXJ0152's FOV are presented in Table 2, and their positions are indicated in the left panel of Figure 4 , mainly lying outside the $z_{s}=6.5$ critical area. For each high- $z$ candidate, we present a magnification estimate (and its statistical uncertainty) from our best-fit model. The absolute magnitude, $M_{\mathrm{uv}}$, at $\lambda=1500 \AA$ is then obtained following the UV continuum slope $f_{\lambda} \propto \lambda^{\beta}$ parameterization for galaxies (Meurer et al. 1999), which we compute with a weighted leastsquares fit using the four WFC3/IR bands (F105W, F125W, F140W, and F160W). The flux corresponding to the redshifted $\lambda=1500 \AA$ is then used to obtain the absolute magnitude, given by $M_{\mathrm{AB}}=31.4-2.5 \log _{10}\left(F_{\mathrm{nJy}}\right)$. As input, we use the EAZY redshift (Brammer et al. 2008) estimates given in Salmon et al. (2017), which consistently predict these objects to be at high $z$ (the scope being to characterize the intrinsic properties of high- $z$ candidates). The resulting rest-frame UV luminosities (corrected for lensing magnifications) have a mean $M_{\mathrm{UV}} \sim-18.2(-19.0)$ and standard deviation $1.03(0.75)$ for the samples at $z=6(7)$, respectively. We used our best-fit SL model to check for (but did not find) any high-redshift multiply imaged galaxies. However, our SL model can provide hints about the true nature of the candidate CL0152-13-0505, which would more probably be a low- $z$ galaxy because, at $z \sim 5.6$, our model predicts further multiple images that we do not identify, whereas for $z \sim 1.0$, a solution predicted by the BPZ photometric code, the galaxy is not multiply imaged.
Another factor that can enhance the lens efficiency of clusters is the high ellipticity or elongation of the lens. The effect of substructures and ellipticity was quantified, for example, in $N$-body simulated and realistic clusters in Meneghetti et al. (2007), where the authors found that substructures and cluster ellipticity account for $\sim 30-40 \%$ of the total cluster cross section, respectively. The combination of these factors explained for instance the unexpectedly high number density of multiple images seen in MACS J0416.12403 at $z=0.40$ (see Zitrin et al. 2013b). The mass distribution of RXJ0152 appears to be highly elongated, for which we estimate an ellipticity (measured as $\left(a^{2}-b^{2}\right)$ / $\left(a^{2}+b^{2}\right)$ ) of $\sim 0.76 \pm 0.02$ in the inner regions but dropping to $\sim 0.54 \pm 0.05$ at larger radius. This value is similar to those measured for MACS J0416.12403 with an ellipticity of $\sim 0.72 \pm 0.01(0.47 \pm 0.04)$ in the inner (outer) regions. These measurements are in agreement with results from $N$-body DMonly simulations (Despali et al. 2017), where $10^{11}-10^{15} M_{\odot} h^{-1}$ halos are more elongated in the center than the outskirts, which still undergo significant interactions with the cluster's environment. In MACS J0416.12403, Zitrin et al. (2013b) identified around 70 multiple images over a critical area of $\sim 0.6 \operatorname{arcmin}^{2}$ (at $z=2$ ) from deeper imaging from the CLASH/HST survey (see Postman et al. 2012). The critical area for $z_{s}=2$ for RXJ0152 is only $\sim 0.1 \operatorname{arcmin}^{2}$, where we find at least 25 multiple images in total (31 if also considering candidate identifications), so the number of multiple images per area, that is, the density of multiple images, is exceptionally high, enabling high-resolution constraints on its central mass distribution.

The enhanced lensing efficiency of RXJ0152 also likely contributes to making the high-redshift, merging cluster RXJ0152 one of the most highly magnifying lenses from the RELICS sample. That said, it should also be noted that cosmic variance can play a nonnegligible role in boosting the number of multiple images and high-redshift galaxies within a cluster's FOV (Leung et al. 2018). The uncertainty in the volume density of highredshift galaxies arising from cosmic variance was estimated to be around $\sim 10-20 \%$ for Lyman-break galaxies at $z \sim 3-4$ (Somerville et al. 2004), but it can increase to $\sim 35 \%$ for higher redshift sources (around $z \sim 5$; Trenti \& Stiavelli 2008). Uncertainties associated with cosmic variance should be carefully taken into account in high-redshift studies, for example, as it can significantly affect the constraints on the faint-end slope of the high-redshift luminosity function (Robertson et al. 2014), which is beyond the scope of this study.

We also note that the rms of our model can be artificially boosted. There are two main reasons for this. The first is technical: the LTM, given it is not fully analytic, is constructed on a grid. The grid's finite resolution (typically similar to or of the order of the HST pixel scale), due to rounding up in the image and source positions, introduces a modest rms boost that can reach $0.1-0.3$ arcsec per system (we now work on assessing this more thoroughly, and more exact results will be reported in future work). The second reason is that the minimum of free parameters and the assumption that mass is coupled to the light distribution, while maximizing prediction power and allowing for the detection of multiple images, do not allow for excessive flexibility in the model, and the fit is limited to the LTM assumption framework. Finally, we would like to emphasize that users should cautiously use SL modeling outputs (such as convergence and magnification) beyond the SL regime where multiple images are seen. The lens model is thus considered an extrapolation beyond this limit. In addition, the smoothing and 
Table 2

High- $z(z \sim 6-7)$ Lensed Candidates

\begin{tabular}{|c|c|c|c|c|c|c|c|c|}
\hline Galaxy ID ${ }^{\mathrm{a}}$ & $\begin{array}{l}\text { R.A. } \\
\text { [J2000] }\end{array}$ & $\begin{array}{c}\text { Decl. } \\
\text { [J2000] }\end{array}$ & $\begin{array}{l}J_{125}{ }^{\mathrm{b}} \\
{[\mathrm{AB}]}\end{array}$ & $z_{\text {phot }}^{\mathrm{BPZc}}$ & $z_{\text {phot }}^{\mathrm{EZ} \mathrm{d}}$ & $\mu_{\mathrm{LTM}}{ }^{\mathrm{e}}$ & $\mu_{\text {Lenstool }} \mathrm{f}$ & $\begin{array}{c}M_{\mathrm{uv}, 1500^{\mathrm{g}}} \\
{[\mathrm{AB}]}\end{array}$ \\
\hline 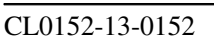 & 28.1748725 & -13.9747007 & $27.21 \pm 0.16$ & $5.9_{-0.3}^{+0.3}$ & $6.1_{-0.4}^{+0.3}$ & $1.45_{-0.04}^{+0.03}$ & $1.33 \pm 0.07$ & $-19.18_{-0.31}^{+0.30}$ \\
\hline CL0152-13-0207 & 28.1713376 & -13.9728768 & $27.37 \pm 0.16$ & $1.0_{-0.2}^{+5.3}$ & $5.7_{-4.5}^{+0.9}$ & $1.88_{-0.13}^{+0.11}$ & $1.53 \pm 0.09$ & $-18.44_{-0.79}^{+0.34}$ \\
\hline CL0152-13-0214 & 28.1733101 & -13.9726804 & $27.52 \pm 0.17$ & $5.5_{-0.5}^{+0.2}$ & $5.7_{-0.7}^{+0.1}$ & $1.74_{-0.14}^{+0.11}$ & $1.49 \pm 0.09$ & $-18.09_{-0.33}^{+0.33}$ \\
\hline CL0152-13-0391 & 28.1999315 & -13.9471136 & $27.15 \pm 0.20$ & $5.6_{-0.3}^{+0.2}$ & $5.8_{-0.4}^{+0.1}$ & $3.73_{-0.65}^{+0.84}$ & $1.29 \pm 0.05$ & $-18.12_{-0.31}^{+0.30}$ \\
\hline CL0152-13-0505 & 28.1838405 & -13.9498063 & $27.70 \pm 0.19$ & $1.1_{-0.2}^{+5.2}$ & $5.6_{-4.6}^{+0.6}$ & $5.49_{-1.48}^{+1.60}$ & $4.09 \pm 1.25$ & $-16.37_{-0.81}^{+0.32}$ \\
\hline CL0152-13-0608 & 28.1785995 & -13.950044 & $27.70 \pm 0.19$ & $5.4_{-0.3}^{+0.3}$ & $5.7_{-0.6}^{+0.2}$ & $2.20_{-0.04}^{+0.04}$ & $1.62 \pm 0.20$ & $-17.45_{-0.32}^{+0.30}$ \\
\hline CL0152-13-0771 & 28.193064 & -13.9545314 & $25.62 \pm 0.06$ & $6.1_{-0.2}^{+0.1}$ & $6.0_{-0.1}^{+0.2}$ & $2.18_{-0.08}^{+0.08}$ & $1.77 \pm 0.35$ & $-20.39_{-0.37}^{+0.41}$ \\
\hline CL0152-13-0800 & 28.1721392 & -13.9550721 & $26.99 \pm 0.13$ & $5.5_{-0.3}^{+0.1}$ & $5.6_{-0.4}^{+0.2}$ & $2.17_{-0.05}^{+0.05}$ & $1.41 \pm 0.12$ & $-18.76_{-0.31}^{+0.30}$ \\
\hline CL0152-13-0924 & 28.197796 & -13.957163 & $27.98 \pm 0.22$ & $5.4_{-4.5}^{+0.2}$ & $5.7_{-1.7}^{+0.1}$ & $2.03_{-0.32}^{+0.20}$ & $1.17 \pm 0.05$ & $-17.73_{-0.41}^{+0.30}$ \\
\hline CL0152-13-1210 & 28.1864788 & -13.9628915 & $27.27 \pm 0.15$ & $6.1_{-5.2}^{+0.4}$ & $5.8_{-4.8}^{+0.9}$ & $1.82_{-0.06}^{+0.06}$ & $1.41 \pm 0.12$ & $-18.44_{-0.82}^{+0.33}$ \\
\hline CL0152-13-1307 & 28.1664822 & -13.9646117 & $27.28 \pm 0.19$ & $5.6_{-4.9}^{+0.3}$ & $5.7_{-4.9}^{+0.4}$ & $5.61_{-1.76}^{+2.05}$ & $1.43 \pm 0.08$ & $-17.13_{-0.85}^{+0.31}$ \\
\hline CL0152-13-1341 & 28.1771307 & -13.9652886 & $28.15 \pm 0.25$ & $0.9_{-0.4}^{+4.7}$ & $5.6_{-5.1}^{+0.3}$ & $3.64_{-0.83}^{+0.90}$ & $2.60 \pm 0.43$ & $-16.89_{-0.93}^{+0.41}$ \\
\hline CL0152-13-1445 & 28.179935 & -13.9671165 & $28.14 \pm 0.25$ & $5.5_{-4.8}^{+0.2}$ & $5.8_{-5.0}^{+0.1}$ & $1.77_{-0.05}^{+0.10}$ & $1.59 \pm 0.16$ & $-17.97_{-0.87}^{+0.35}$ \\
\hline CL0152-13-1494 & 28.1869999 & -13.9682532 & $27.53 \pm 0.18$ & $5.6_{-4.8}^{+0.3}$ & $5.8_{-5.1}^{+0.2}$ & $1.41_{-0.03}^{+0.03}$ & $1.21 \pm 0.06$ & $-18.41_{-0.87}^{+0.30}$ \\
\hline CL0152-13-1508 & 28.1656067 & -13.9686442 & $24.54 \pm 0.04$ & $5.6_{-0.1}^{+0.1}$ & $5.5_{-0.2}^{+0.3}$ & $3.54_{-0.09}^{+0.10}$ & $1.47 \pm 0.07$ & $-20.35_{-0.30}^{+0.31}$ \\
\hline CL0152-13-1546 & 28.1782025 & -13.9693313 & $28.04 \pm 0.21$ & $5.4_{-4.8}^{+0.4}$ & $5.7_{-4.8}^{+0.3}$ & $1.67_{-0.05}^{+0.04}$ & $1.51 \pm 0.12$ & $-18.09_{-0.83}^{+0.30}$ \\
\hline CL0152-13-1569 & 28.1779564 & -13.9696918 & $28.15 \pm 0.25$ & $0.9_{-0.3}^{+4.6}$ & $5.6_{-4.6}^{+0.6}$ & $1.65_{-0.05}^{+0.04}$ & $1.49 \pm 0.12$ & $-18.19_{-0.81}^{+0.30}$ \\
\hline CL0152- 13-1576 & 28.1816193 & -13.9699027 & $27.15 \pm 0.14$ & $5.5_{-5.0}^{+0.2}$ & $5.5_{-4.9}^{+0.4}$ & $1.47_{-0.04}^{+0.03}$ & $1.31 \pm 0.08$ & $-18.79_{-0.87}^{+0.31}$ \\
\hline CL0152-13-1642 & 28.1881258 & -13.9439934 & $27.95 \pm 0.22$ & $5.8_{-4.8}^{+0.4}$ & $5.9_{-4.9}^{+0.6}$ & $5.14_{-2.05}^{+1.30}$ & $1.94 \pm 0.17$ & $-16.85_{-0.83}^{+0.32}$ \\
\hline CL0152-13-0191 & 28.1716411 & -13.9734429 & $27.13 \pm 0.28$ & $6.6_{-0.5}^{+0.5}$ & $6.9_{-0.7}^{+0.6}$ & $1.78_{-0.08}^{+0.06}$ & $1.51 \pm 0.08$ & $-19.10_{-0.32}^{+0.31}$ \\
\hline CL0152-13-0259 & 28.1825175 & -13.9717095 & $26.36 \pm 0.23$ & $6.7_{-0.4}^{+0.4}$ & $7.0_{-0.5}^{+0.5}$ & $1.40_{-0.03}^{+0.02}$ & $1.24 \pm 0.07$ & $-20.16_{-0.31}^{+0.31}$ \\
\hline CL0152-13-0410 & 28.1824016 & -13.9469211 & $26.58 \pm 0.24$ & $6.4_{-0.3}^{+0.2}$ & $6.7_{-0.4}^{+0.2}$ & $2.58_{-0.05}^{+0.04}$ & $1.75 \pm 0.24$ & $-19.24_{-0.31}^{+0.30}$ \\
\hline CL0152- 13-0525 & 28.1926776 & -13.9499311 & $27.19 \pm 0.29$ & $6.8_{-5.8}^{+0.7}$ & $7.2_{-5.9}^{+0.8}$ & $4.02_{-0.10}^{+0.11}$ & $1.83 \pm 0.16$ & $-18.02_{-0.84}^{+0.32}$ \\
\hline CL0152-13-1254 & 28.1806823 & -13.9635242 & $27.20 \pm 0.28$ & $6.5_{-5.5}^{+0.4}$ & $6.9_{-5.6}^{+0.3}$ & $2.56_{-0.33}^{+0.42}$ & $2.24 \pm 0.40$ & $-18.31_{-0.83}^{+0.32}$ \\
\hline
\end{tabular}

Notes.

${ }^{a}$ Galaxy ID, following Salmon et al. (2017) notations. Note that the cluster is also named CL0152.7-1357. The horizontal line separates candidates at $z \sim 6$ and $z \sim 7$.

${ }^{\mathrm{b}}$ Apparent magnitude in the F125W band.

${ }^{\mathrm{c}}$ Redshift estimation based on the BPZ pipeline along with their $1 \sigma$ uncertainties.

${ }^{\mathrm{d}}$ Redshift estimation based on the EAZY pipeline along with their $1 \sigma$ uncertainties.

${ }^{\mathrm{e}}$ Magnification estimates (at the respective source redshift) from our LTM best-fit model and the corresponding statistical uncertainty (measured as the standard deviation). The best-fit value is the one used for all relevant computations.

${ }^{\mathrm{f}}$ Average magnification estimates (at the respective source redshift) from our Lenstool model and statistical uncertainty (i.e., the standard deviation) from 2000 MCMC models.

$\mathrm{g}$ Absolute magnitude, $M_{\mathrm{uv}}$, at $\lambda=1500 \AA$ for which the errors have been propagated from the photometric and magnification uncertainties based on our best-fit LTM model.

other interpolations used in our methodology can introduce artifacts at the edges of the modeled FOV.

Recent studies have focused on better understanding and quantifying the impact of systematic errors arising from different assumptions (i.e., different algorithms) in the modeling of strong lensing clusters (Treu et al. 2016; Bouwens et al. 2017; Meneghetti et al. 2017). We have then modeled RXJ0152 with the Lenstool in order to compare the main SL outputs between the two modeling tools that are the main algorithms providing SL models of RELICS clusters for the community.

We find that the resulting mass distributions are in good agreement, as shown in Figure 3. While the Lenstool model estimates a higher mass in the inner region of the cluster core (the LTM being shallower, as is typically the case) and the LTM model is more massive in the outskirts due to the structure in the $\mathrm{SW}$, both models are in very good agreement in the intermediate region.

Regarding the high- $z$ candidates, the magnification estimates are in fairly good agreement between the two models, but the discrepancies between models become larger for high magnification values (Bouwens et al. 2017). The LTM pipeline predicts some high-redshift candidates with significantly higher magnification values. This is partly due to some highmagnification regions between the merging clumps that we did not include in the Lenstool model.

\section{Summary}

The merging galaxy cluster RXJ0152.7-1357 (also known as CL0152.7-1357; $z=0.83$ ) is one of the X-ray-brightest and thus best-studied clusters at such high redshifts, but it is missing a full, public SL analysis to date.

In this work, we have presented an SL analysis of RXJ0152 based on recent observations from the RELICS survey and adopting a light-traces-mass methodology that allowed us to uncover several sets of multiple images of background galaxies to be used as constraints for the modeling. Umetsu et al. (2005) had uncovered and spectroscopically confirmed one multiply imaged system that allowed them to put constraints on the NE clump of the cluster. Thanks to the RELICS survey, we were 
now able to uncover a relatively large number of new multiple images over a small area of just $\sim 0.4 \operatorname{arcmin}^{2}$, allowing us to put high-resolution constraints on the central mass distribution of RXJ0152.7-1357.

The mass distribution of RXJ0152's core, as revealed by our SL modeling and as indicated by the member galaxy distribution, shows a clumpy morphology made of several substructures, which further supports the merging scenario reported in previous works (Maughan et al. 2003; Jee et al. 2005a). RXJ0152 appears to be a modest lens with relatively small critical curves for a source redshift $z \sim 2$, over several merging clumps, and enclosing a mass of $(2.5 \pm 0.4) \times 10^{13} M_{\odot}$. We note that, together with the Baby Bullet cluster, RXJ0152 was one of the two RELICS clusters not detected in the SZ mass Planck cluster catalog PSZ2 (Planck Collaboration et al. 2015). For higher redshift sources $(z \sim 9)$, these critical curves merge, boosting the critical area. As a result, RXJ0152.7-1357 presents a slightly smaller than but overall similar lensing strength for $z \sim 9$ sources to other RELICS clusters that were found to be massive and prominent lenses (see Figure 4).

The elongated substructure chain composing RXJ0152 results in an efficient lens (e.g., Merten et al. 2011; Limousin et al. 2012), accounting in part for the high density of multiply imaged galaxies. RXJ0152 also comprises the third-largest sample of high-redshift $(z \sim 6-7)$ candidates among all clusters in the RELICS program (Salmon et al. 2017), a sample that we further characterized in this work thanks to our lens model. This RELICS cluster shows the advantage of targeting highredshift, merging clusters, even if modestly massive, as the high level of the substructure, together with its elongated shape, boosts the lensing efficiency (Zitrin et al. 2013a).

Finally, we present a comparison between the LTM and Lenstool SL models. In general, both the mass distribution and magnification values are in good agreement, the differences arising from the distinct assumptions in the modeling techniques and parameterization. The LTM modeling estimates a larger high-magnification area due to the SW structure. A more detailed comparison between these two techniques is relegated to a future paper.

Among all massive galaxy clusters observed with $H S T$ to date, there are a significant number of clusters having none or few spectroscopically measured multiple images. Similarly,
RXJ0152 has only one spectroscopically measured system, presented in Umetsu et al. (2005). Upcoming observing campaigns will help overcome this source of systematic uncertainties, probably the main caveat of current SL models (Johnson \& Sharon 2016; Remolina González et al. 2018).

The lens models presented in this work, as well as magnification maps, are made publicly available through the MAST archive (see footnote 20).

We kindly thank the anonymous referee for suggestions that helped to improve the paper. A.A. thanks Jean-Paul Kneib and Eric Jullo for useful discussions. This work is based on observations taken by the RELICS Treasury Program (GO14096) with the NASA/ESA HST. Program GO-14096 is supported by NASA through a grant from the Space Telescope Science Institute, which is operated by the Association of Universities for Research in Astronomy, Inc., under NASA contract NAS5-26555. This work was performed in part under the auspices of the U.S. Department of Energy by Lawrence Livermore National Laboratory under contract DE-AC5207NA27344. This paper includes data gathered with the 6.5 meter Magellan Telescopes located at Las Campanas Observatory, Chile. We thank Ian Roederer for executing the Magellan observation. K.U. acknowledges support from the Ministry of Science and Technology of Taiwan under grant MOST 1062628-M-001-003-MY3. R.C.L. acknowledges support from an Australian Research Council Discovery Early Career Researcher Award (DE180101240). This work is partially supported by the Australian Research Council Centre of Excellence for All-Sky Astrophysics in 3 Dimensions (ASTRO-3D). S.T. acknowledges support from the ERC Consolidator Grant funding scheme (project ConTExt, grant No. 648179). The Cosmic Dawn Center is funded by the Danish National Research Foundation.

\section{Appendix A Reproduction of Multiple Images}

The multiple image reproductions from the LTM best-fit model are shown in Figure 5. 
1.1
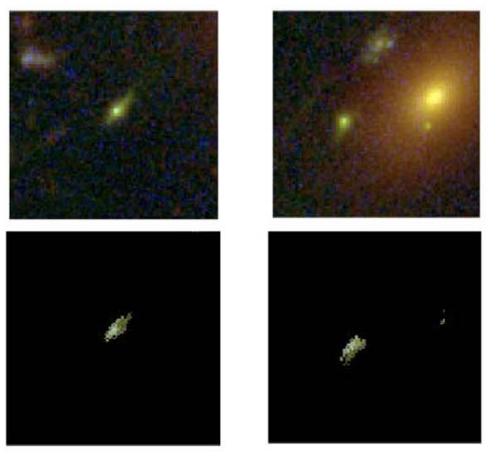

$3.2+4.2$
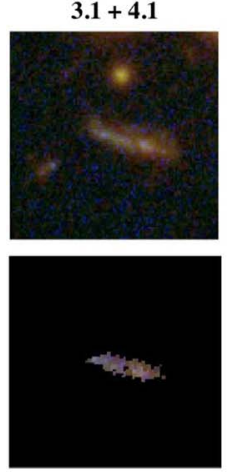

6.1
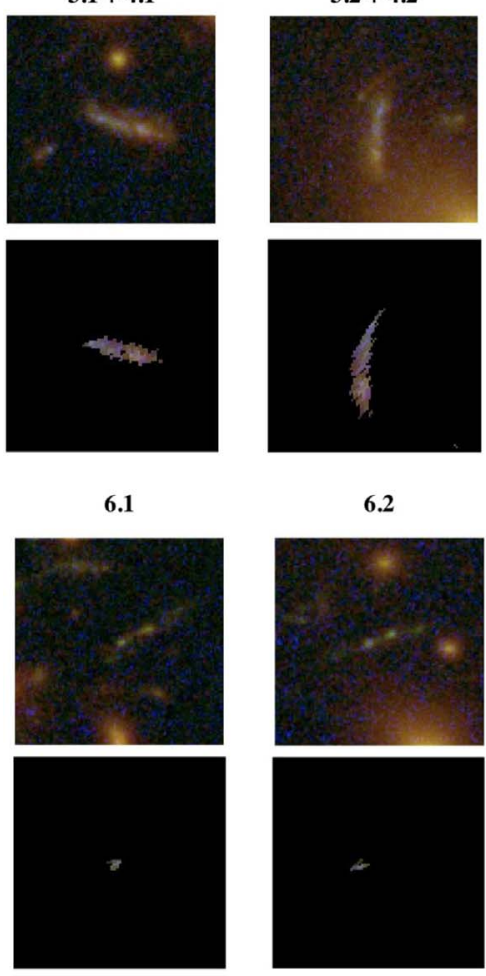

6.2
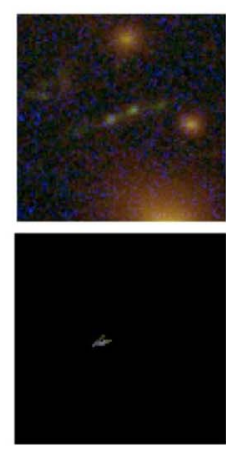

1.3
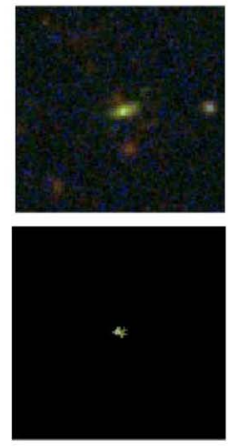

2.1
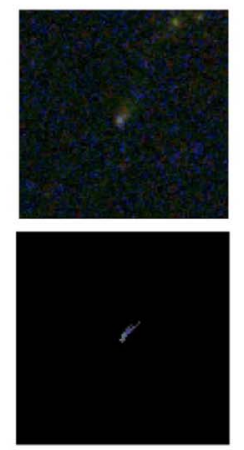

$3.3+4.3$
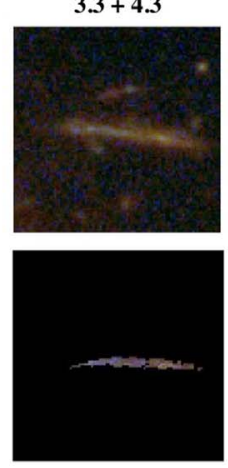

6.3
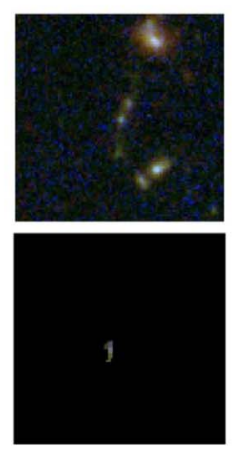

7.1
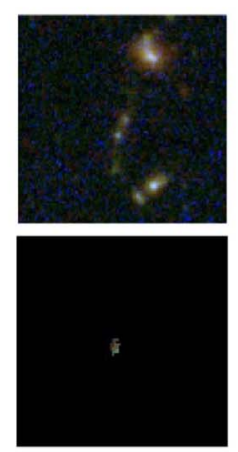
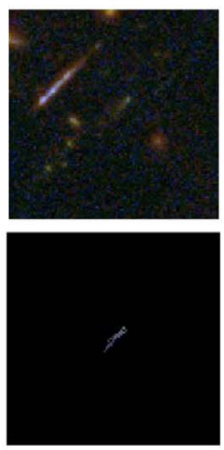

2.3
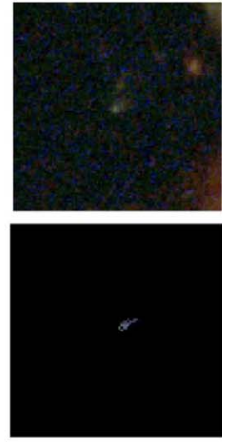

$5.1+5.2$
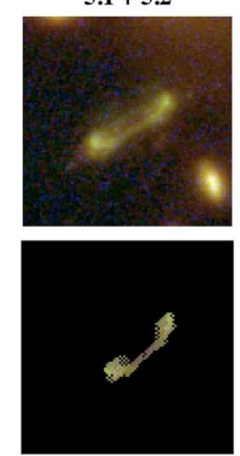

7.2
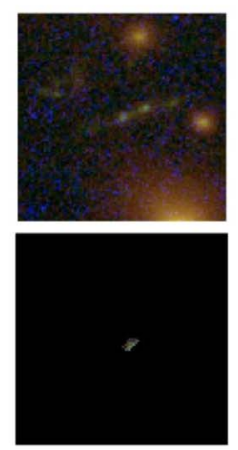

7.3
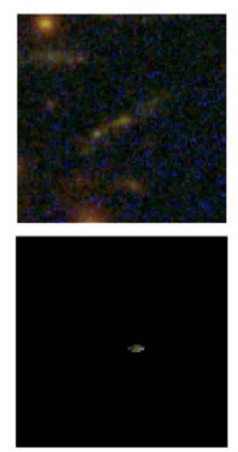

8.1
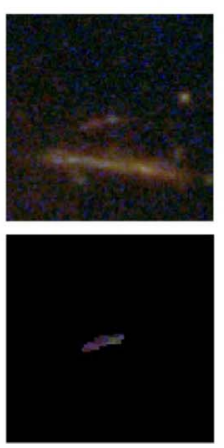

8.2
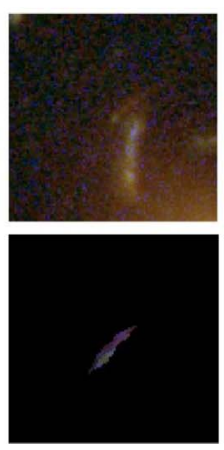

8.3
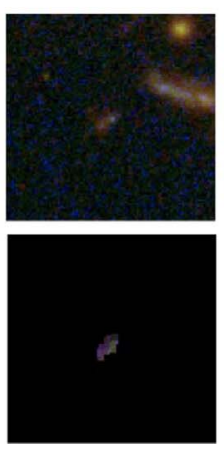

Figure 5. Reproduction of multiple images by our best-fit LTM model for RXJ0152. For each image, we delens the first image of the system to the source plane and back to the image plane to be compared to the other images of that system. The orientation and internal details of the model-predicted images (bottom rows) are similar to those of the observed images (upper rows). 


\section{Appendix B}

\section{RXJ0152's Lenstool Model}

Additional details regarding the results from the SL modeling of RXJ0152 are given in Figures 6 and 7.

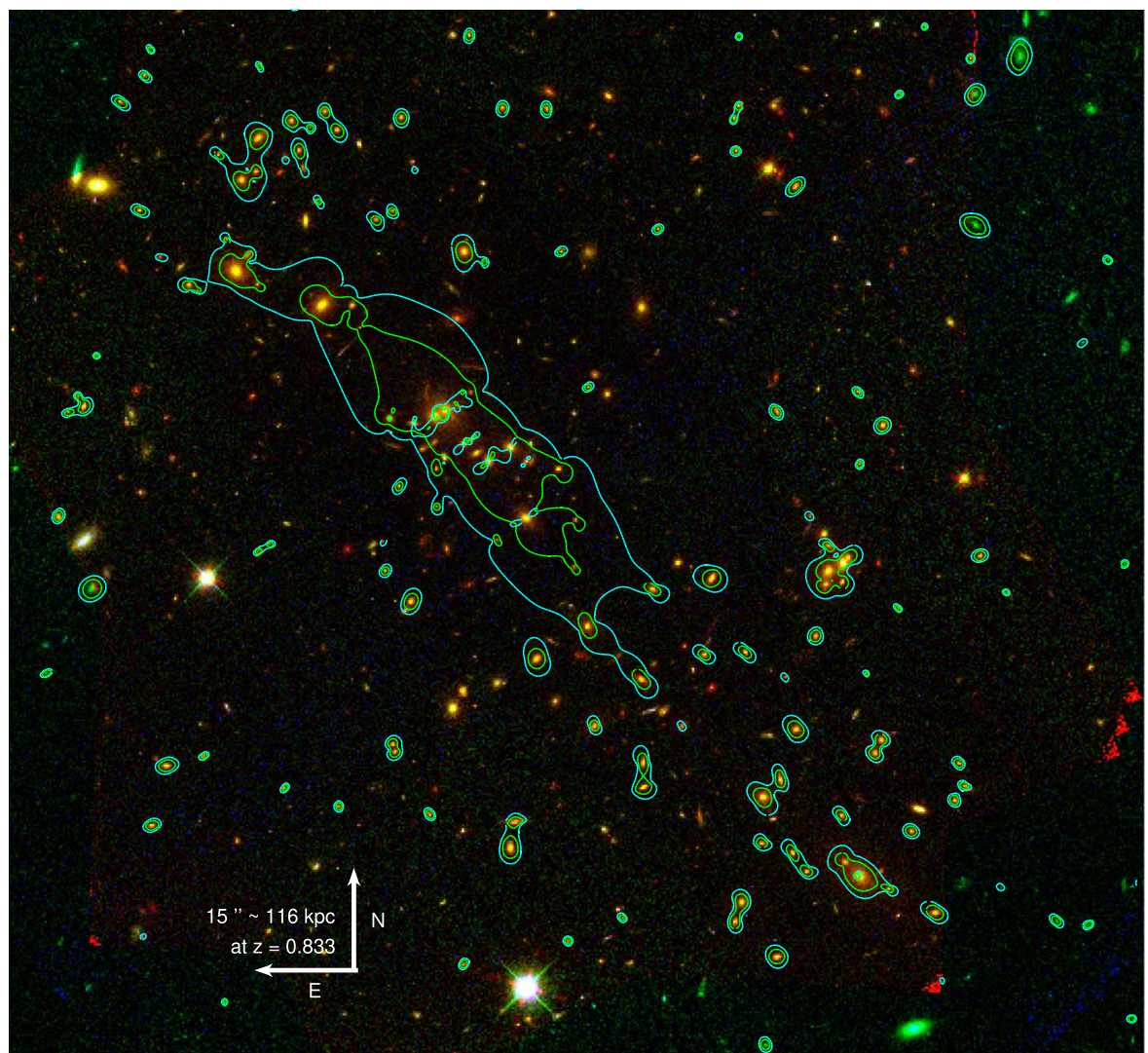

Figure 6. Critical curves from our best-fit Lenstool model at redshifts $z_{s}=2$ and $z_{s}=9$ in green and cyan, respectively.

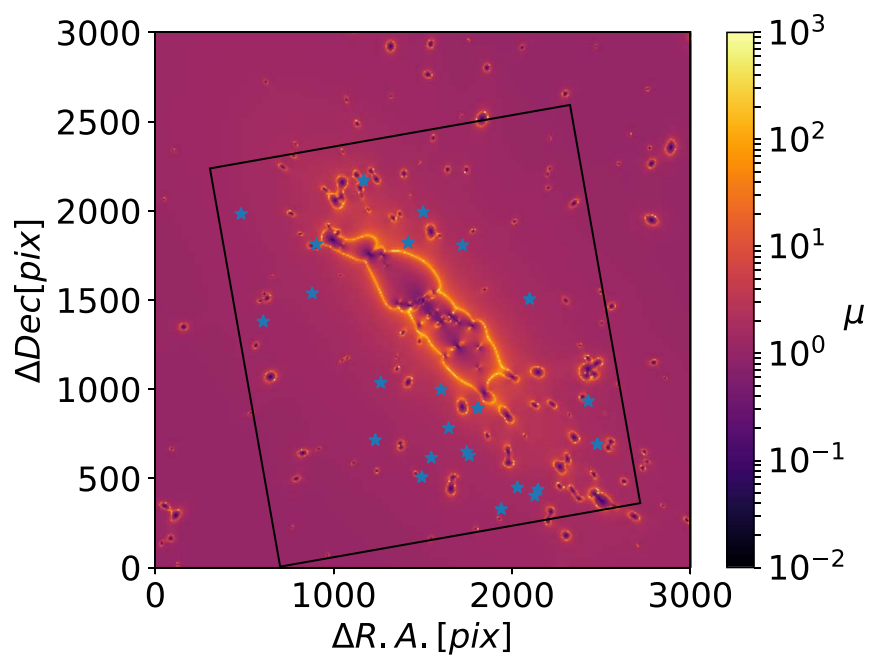

Figure 7. Magnification map from our best-fit Lenstool model for a source at $z_{s}=6.5$. Same symbols as in Figure 4 .

\section{Appendix C}

\section{Best-fit Parameters}

Additional details regarding the SL modelings performed with LTM and Lenstool are given in Tables 3 and 4.
Table 3

Best-fit Parameters from the LTM Model

\begin{tabular}{lcccccc}
\hline \hline Component & $q^{\mathrm{a}}$ & $s^{\mathrm{a}}$ & $k_{\text {new }}{ }^{\mathrm{a}}$ & $k_{\text {gal }}{ }^{\mathrm{a}}$ & $\gamma^{\mathrm{b}}$ & $\phi^{\mathrm{b}}$ \\
\hline $\begin{array}{l}\text { Total mass } \\
\text { distribution }\end{array}$ & $1.40_{-0.04}^{+0.03}$ & $88.0_{-6.0}^{+7.0}$ & $1.21_{-0.10}^{+0.09}$ & $0.15_{-0.03}^{+0.04}$ & $\ldots$ & $\cdots$ \\
\hline External shear & $\ldots$ & $\ldots$ & $\ldots$ & $\ldots$ & $0.05_{-0.04}^{+0.4}$ & $0.50_{-0.37}^{+0.35}$ \\
\hline
\end{tabular}

Notes.

${ }^{\text {a }}$ Best-fit values with $1 \sigma$ uncertainties of the basic LTM parameters presented in Section 3.1.

${ }^{\mathrm{b}}$ Amplitude and position angle of the external shear with $1 \sigma$ uncertainties.

Table 4

Best-fit Parameters from the Lenstool Model

\begin{tabular}{lccccccc}
\hline \hline Component & $\begin{array}{c}\Delta \alpha^{\mathrm{a}} \\
\left({ }^{\prime \prime}\right)\end{array}$ & $\begin{array}{c}\Delta \delta^{\mathrm{a}} \\
\left({ }^{\prime \prime}\right)\end{array}$ & $\varepsilon^{\mathrm{b}}$ & $\begin{array}{c}\theta^{\mathrm{c}} \\
(\mathrm{deg})\end{array}$ & $\begin{array}{c}\sigma_{0}{ }^{\mathrm{d}} \\
\left(\mathrm{km} \mathrm{s}^{-1}\right)\end{array}$ & $\begin{array}{c}\mathrm{r}_{\text {cut }}{ }^{\mathrm{d}} \\
(\mathrm{kpc})\end{array}$ & $\begin{array}{c}\mathrm{r}_{\text {core }}{ }^{\mathrm{d}} \\
(\mathrm{kpc})\end{array}$ \\
\hline $\mathrm{DM}$ & $3.7_{-1.1}^{+2.0}$ & $-4.7_{-2.0}^{+1.5}$ & $0.74_{-0.08}^{+0.04}$ & $130.0_{-1.2}^{+1.2}$ & $926.1_{-40.8}^{+41.1}$ & {$[1500.0]^{\mathrm{e}}$} & $8.7_{-1.7}^{+2.5}$ \\
$L^{*}$ Galaxy & $\ldots$ & $\ldots$ & $\ldots$ & $\ldots$ & $276.5_{-19.4}^{+18.0}$ & $\ldots$ & $\ldots$
\end{tabular}

Notes.

${ }^{\text {a }}$ Positional offsets with respect to the reference point (R.A $=28.183021 \mathrm{deg}$; decl. $=-13.955764 \mathrm{deg}$ ).

b Ellipticity.

c Position angle.

${ }^{\mathrm{d}}$ PIEMD best-fit parameters.

${ }^{\mathrm{e}}$ Fixed value. 


\section{ORCID iDs}

Ana Acebron (10) https://orcid.org/0000-0003-3108-9039 Adi Zitrin (10 https://orcid.org/0000-0002-0350-4488

Guillaume Mahler (iD https://orcid.org/0000-0003-3266-2001

Dan Coe (iD https://orcid.org/0000-0001-7410-7669

Keren Sharon (iD https://orcid.org/0000-0002-7559-0864

Nathália Cibirka (iD https://orcid.org/0000-0002-2356-4680

Maruša Bradač (i) https://orcid.org/0000-0001-5984-0395

Michele Trenti (i) https://orcid.org/0000-0001-9391-305X

Keiichi Umetsu (iD https://orcid.org/0000-0002-7196-4822

Felipe Andrade-Santos (i) https://orcid.org/0000-0002-

8144-9285

Larry Bradley (iD https://orcid.org/0000-0002-7908-9284

Daniela Carrasco (D) https://orcid.org/0000-0002-3772-0330

William A. Dawson (iD https://orcid.org/0000-0003-0248-6123

Austin T. Hoag (D) https://orcid.org/0000-0001-8989-2567

Kuang-Han Huang (D) https://orcid.org/0000-0001-7826-6448

Traci L. Johnson (1D https://orcid.org/0000-0002-8829-5303

Daniel Lam (1) https://orcid.org/0000-0002-6536-5575

Rachael C. Livermore (ib https://orcid.org/0000-00034456-1566

Ramesh Mainali (10 https://orcid.org/0000-0003-0094-6827

Pascal A. Oesch (1) https://orcid.org/0000-0001-5851-6649

Masami Ouchi (iD https://orcid.org/0000-0002-1049-6658

Rachel Paterno-Mahler (i) https://orcid.org/0000-0003-

3653-3741

Brett Salmon (iD https://orcid.org/0000-0002-7453-7279

Victoria Strait (iD https://orcid.org/0000-0002-6338-7295

Sune Toft (iD https://orcid.org/0000-0003-3631-7176

Benedetta Vulcani (i) https://orcid.org/0000-0003-0980-1499

\section{References}

Acebron, A., Cibirka, N., Zitrin, A., et al. 2018, ApJ, 858, 42 Atek, H., Richard, J., Jauzac, M., et al. 2015, ApJ, 814, 69 Benítez, N. 2000, ApJ, 536, 571

Benítez, N., Ford, H., Bouwens, R., et al. 2004, ApJS, 150, 1

Bertin, E., \& Arnouts, S. 1996, A\&AS, 117, 393

Boselli, A., \& Gavazzi, G. 2006, PASP, 118, 517

Bouwens, R. J., Oesch, P. A., Illingworth, G. D., Ellis, R. S., \& Stefanon, M. 2017, ApJ, 843, 129

Bradač, M., Allen, S. W., Treu, T., et al. 2008, ApJ, 687, 959

Brammer, G. B., van Dokkum, P. G., \& Coppi, P. 2008, ApJ, 686, 1503

Broadhurst, T., Takada, M., Umetsu, K., et al. 2005, ApJL, 619, L143

Cerny, C., Sharon, K., Andrade-Santos, F., et al. 2018, ApJ, 859, 159

Cibirka, N., Acebron, A., Zitrin, A., et al. 2018, ApJ, 863, 145

Clowe, D., Bradač, M., Gonzalez, A. H., et al. 2006, ApJL, 648, L109

Coe, D., Benítez, N., Sánchez, S. F., et al. 2006, AJ, 132, 926

Coe, D., Salmon, B., Bradac, M., et al. 2019, ApJ, submitted, arXiv:1903.02002

Coe, D., Zitrin, A., Carrasco, M., et al. 2013, ApJ, 762, 32

Coogan, R. T., Daddi, E., Sargent, M. T., et al. 2018, MNRAS, 479, 703

Dawson, W. A., Wittman, D., Jee, M. J., et al. 2012, ApJL, 747, L42

Della Ceca, R., Scaramella, R., Gioia, I. M., et al. 2000, A\&A, 353, 498

Demarco, R., Gobat, R., Rosati, P., et al. 2010, ApJ, 725, 1252

Demarco, R., Rosati, P., Lidman, C., et al. 2005, A\&A, 432, 381

Deshev, B., Finoguenov, A., Verdugo, M., et al. 2017, A\&A, 607, A131

Despali, G., Giocoli, C., Bonamigo, M., Limousin, M., \& Tormen, G. 2017, MNRAS, 466, 181
Dressler, A., Bigelow, B., Hare, T., et al. 2011, PASP, 123, 288

Ebeling, H., Jones, L. R., Perlman, E., et al. 2000, ApJ, 534, 133

Faber, S. M., \& Jackson, R. E. 1976, ApJ, 204, 668

Fedeli, C., Meneghetti, M., Gottlöber, S., \& Yepes, G. 2010, A\&A, 519, A91

Frye, B. L., Pascale, M., Zitrin, A., et al. 2019, ApJ, 871, 51

Girardi, M., Demarco, R., Rosati, P., \& Borgani, S. 2005, A\&A, 442, 29

Gladders, M. D., \& Yee, H. K. C. 2000, AJ, 120, 2148

Grillo, C., Suyu, S. H., Rosati, P., et al. 2015, ApJ, 800, 38

Hashimoto, T., Laporte, N., Mawatari, K., et al. 2018, Natur, 557, 392

Huo, Z.-Y., Xue, S.-J., Xu, H., Squires, G., \& Rosati, P. 2004, AJ, 127, 1263

Jauzac, M., Richard, J., Jullo, E., et al. 2015, MNRAS, 452, 1437

Jee, M. J., White, R. L., Benítez, N., et al. 2005a, ApJ, 618, 46

Jee, M. J., White, R. L., Ford, H. C., et al. 2005b, ApJ, 634, 813

Johnson, T. L., \& Sharon, K. 2016, ApJ, 832, 82

Jørgensen, I., Bergmann, M., Davies, R., et al. 2005, AJ, 129, 1249

Joy, M., LaRoque, S., Grego, L., et al. 2001, ApJL, 551, L1

Jullo, E., Kneib, J.-P., Limousin, M., et al. 2007, NJPh, 9, 447

Kassiola, A., \& Kovner, I. 1993, ApJ, 417, 450

Khullar, G., Bleem, L. E., Bayliss, M. B., et al. 2019, ApJ, 870, 7

Krause, E., Pierpaoli, E., Dolag, K., \& Borgani, S. 2012, MNRAS, 419, 1766

Leung, E., Broadhurst, T., Lim, J., et al. 2018, ApJ, 862, 156

Limousin, M., Ebeling, H., Richard, J., et al. 2012, A\&A, 544, A71

Limousin, M., Richard, J., Jullo, E., et al. 2016, A\&A, 588, A99

Livermore, R. C., Finkelstein, S. L., \& Lotz, J. M. 2017, ApJ, 835, 113

Lotz, J. M., Koekemoer, A., Coe, D., et al. 2017, ApJ, 837, 97

Markevitch, M., Gonzalez, A. H., David, L., et al. 2002, ApJL, 567, L27

Maughan, B. J., Jones, L. R., Ebeling, H., et al. 2003, ApJ, 587, 589

Meneghetti, M., Argazzi, R., Pace, F., et al. 2007, A\&A, 461, 25

Meneghetti, M., Natarajan, P., Coe, D., et al. 2017, MNRAS, 472, 3177

Merten, J., Coe, D., Dupke, R., et al. 2011, MNRAS, 417, 333

Meurer, G. R., Heckman, T. M., \& Calzetti, D. 1999, ApJ, 521, 64

Monna, A., Seitz, S., Balestra, I., et al. 2017, MNRAS, 466, 4094

Oemler, A., Clardy, K., Kelson, D., Walth, G., \& Villanueva, E. 2017, COSMOS: Carnegie Observatories System for MultiObject Spectroscopy, Astrophysics Source Code Library, ascl:1705.001

Paterno-Mahler, R., Sharon, K., Coe, D., et al. 2018, ApJ, 863, 154

Peebles, P. J. E., Daly, R. A., \& Juszkiewicz, R. 1989, ApJ, 347, 563

Planck Collaboration, Ade, P. A. R., Aghanim, N., et al. 2015, A\&A, 582, A29

Planck Collaboration, Ade, P. A. R., Aghanim, N., et al. 2016, A\&A, 594, A27

Poole, G. B., Babul, A., McCarthy, I. G., et al. 2007, MNRAS, 380, 437

Postman, M., Coe, D., Benítez, N., et al. 2012, ApJS, 199, 25

Redlich, M., Bartelmann, M., Waizmann, J.-C., \& Fedeli, C. 2012, A\&A, 547, A66

Remolina González, J. D., Sharon, K., \& Mahler, G. 2018, ApJ, 863, 60

Richard, J., Kneib, J.-P., Limousin, M., Edge, A., \& Jullo, E. 2010, MNRAS, 402, L44

Richard, J., Stark, D. P., Ellis, R. S., et al. 2008, ApJ, 685, 705

Robertson, B. E., Ellis, R. S., Dunlop, J. S., et al. 2014, ApJL, 796, L27

Romer, A. K., Nichol, R. C., Holden, B. P., et al. 2000, ApJS, 126, 209

Rosati, P., Della Ceca, R., Norman, C., \& Giacconi, R. 1998, ApJL, 492, L21

Salmon, B., Coe, D., Bradley, L., et al. 2017, ApJ, submitted, arXiv:1710. 08930

Somerville, R. S., Lee, K., Ferguson, H. C., et al. 2004, ApJL, 600, L171

Tanaka, M., Kodama, T., Arimoto, N., \& Tanaka, I. 2006, MNRAS, 365, 1392

Torri, E., Meneghetti, M., Bartelmann, M., et al. 2004, MNRAS, 349, 476

Trenti, M., \& Stiavelli, M. 2008, ApJ, 676, 767

Treu, T., Brammer, G., Diego, J. M., et al. 2016, ApJ, 817, 60

Umetsu, K., Tanaka, M., Kodama, T., et al. 2005, PASJ, 57, 877

Wong, K. C., Ammons, S. M., Keeton, C. R., \& Zabludoff, A. I. 2012, ApJ, 752,104

Zheng, W., Postman, M., Zitrin, A., et al. 2012, Natur, 489, 406

Zitrin, A., Broadhurst, T., Umetsu, K., et al. 2009, MNRAS, 396, 1985

Zitrin, A., Fabris, A., Merten, J., et al. 2015, ApJ, 801, 44

Zitrin, A., Menanteau, F., Hughes, J. P., et al. 2013a, ApJL, 770, L15

Zitrin, A., Meneghetti, M., Umetsu, K., et al. 2013b, ApJL, 762, L30 